\title{
NEW RADIO OBSERVATIONS OF ANOMALOUS MICROWAVE EMISSION IN THE H II REGION RCW175
}

\author{
E. S. Battistelli ${ }^{1}$, E. Carretti ${ }^{2}$, A. Cruciani ${ }^{1,3}$, P. De Bernardis ${ }^{1}$, R. Génova-Santos ${ }^{4,5}$, S. Masi $^{1}$,

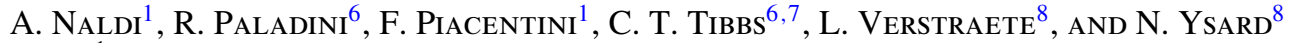 \\ ${ }^{1}$ Department of Physics, Sapienza University of Rome, Piazzale Aldo Moro 5, I-00185 Rome, Italy \\ ${ }^{2}$ CSIRO Astronomy and Space Science, P.O. Box 76, Epping, NSW 1710, Australia \\ 3 INFN Sezione di Roma, I-00185 Rome, Italy \\ ${ }^{4}$ Instituto de Astrofisica de Canarias, C/Vía Lactea, s/n, La Laguna (Tenerife) E-38205, Spain \\ ${ }^{5}$ Departmento de Astrofisica, Universidad de La Laguna (ULL), 38206 La Leguna, Tenerife, Spain \\ ${ }^{6}$ Infrared Processing Analysis Center, California Institute of Technology, Pasadena, CA 91125, USA \\ ${ }^{7}$ Scientific Support Office, Directorate of Science and Robotic Exploration, European Space Research and Technology Centre \\ (ESA/ESTEC), Keplerlaan 1, 2201 AZ Noordwijk, The Netherlands \\ ${ }^{8}$ IAS, CNRS (UMR 8617), Université Paris-Sud 11, bâtiment 121, F-91400 Orsay, France \\ Received 2014 June 11; accepted 2015 January 9; published 2015 March 12
}

\begin{abstract}
We have observed the $\mathrm{H}$ II region RCW 175 with the $64 \mathrm{~m}$ Parkes telescope at $8.4 \mathrm{GHz}$ and $13.5 \mathrm{GHz}$ in total intensity, and at $21.5 \mathrm{GHz}$ in both total intensity and polarization. High angular resolution ranging from 1 to $2.4 \mathrm{arcmin}$, high sensitivity, and polarization capability enable us to perform a detailed study of the different constituents of the $\mathrm{H}$ II region. For the first time, we resolve three distinct regions at microwave frequencies, two of which are part of the same annular diffuse structure. Our observations enable us to confirm the presence of anomalous microwave emission (AME) from RCW175. Fitting the integrated flux density across the entire region with the currently available spinning dust models, using physically motivated assumptions, indicates the presence of at least two spinning dust components: a warm component $\left(T_{\text {gas }}=5800 \mathrm{~K}\right)$ with a relatively large hydrogen number density $n_{\mathrm{H}}=26.3 / \mathrm{cm}^{3}$ and a cold component $\left(T_{\text {gas }}=100 \mathrm{~K}\right)$ with a hydrogen number density of $n_{\mathrm{H}}=150 / \mathrm{cm}^{3}$. The present study is an example highlighting the potential of using high angular-resolution microwave data to break model parameter degeneracies. Thanks to the spectral coverage and angular resolution of the Parkes observations, we have been able to derive one of the first AME/excess maps, at $13.5 \mathrm{GHz}$, showing clear evidence that the bulk of the anomalous emission arises in particular from one of the source components, with some additional contribution from the diffuse structure. A cross-correlation analysis with thermal dust emission has shown a high degree of correlation with one of the regions within RCW175. In the center of RCW175, we find an average polarized emission at $21.5 \mathrm{GHz}$ of $2.2 \pm 0.2$ (rand.) \pm 0.3 (sys.) \% of the total emission, where we have included both systematic and statistical uncertainties at $68 \% \mathrm{CL}$. This polarized emission could be due to sub-dominant synchrotron emission from the region and is thus consistent with very faint or non-polarized emission associated with AME.
\end{abstract}

Key words: dust, extinction - H II regions - methods: data analysis - polarization - radiation mechanisms: general radio continuum: ISM

\section{INTRODUCTION}

$\mathrm{H}$ II regions play a crucial role in broadening our understanding of the mechanisms regulating the interstellar medium (ISM). The complex morphology of $\mathrm{H}$ II regions is the result of an ensemble of factors, i.e., the evolutionary stage, the number and spectral type of the ionizing sources, and their interplay with the surrounding medium. RCW175 is a relatively well-studied, complex H II region, and is approximately $10 \times 15 \mathrm{arcmin}^{2}$ in size. The region was identified by Sharpless (1959) and has been observed at various wavelengths ever since. In the microwave range it was first observed by the Very Small Array (VSA) experiment (Todorovic et al. 2010), which found evidence of an anomalous microwave emission (AME) excess, and then by the Cosmic Background Imager (CBI) experiment, which confirmed the anomalous excess and a basic structure of at least two components at $\sim 30 \mathrm{GHz}$ (Dickinson et al. 2009).

AME was first identified as diffuse emission in the $10-60 \mathrm{GHz}$ range in excess of the expected level of microwave emission from free-free, synchrotron, and thermal dust emission by experiments such as the Cosmic Background Explorer-DMR (Kogut et al. 1996), the Saskatoon experiment (de OliveiraCosta et al. 1997), Owens Valley Radio Observatory (Leitch et al. 1997), and the Tenerife experiment (de Oliveira-Costa et al. 1999). Several authors have found further statistical evidence of AME (see, e.g., Banday et al. 2003; Lagache 2003; de Oliveira-Costa et al. 2004; Finkbeiner et al. 2004; Davies et al. 2006; Hildebrandt et al. 2007; Lu et al. 2012; MivilleDeschênes et al. 2008), while it has also been detected directly in individual regions in a limited number of cases (see, e.g., Watson et al. 2005; Scaife et al. 2007; Casassus et al. 2008; AMI Consortium 2009; Murphy et al. 2010; Dickinson et al. 2010; Genova-Santos et al. 2011; Planck Collaboration 2011a, 2014a). Recent models predict that the AME is dominated by electric dipole emission from the smallest grains, possibly polycyclic aromatic hydrocarbons (PAHs; Draine \& Lazarian 1998; Ysard et al. 2010; Hoang et al. 2010). However, other physical emission mechanisms, such as hot free-free emission (Leitch et al. 1997), hard synchrotron radiation (Bennett et al. 2003), or magnetic dipole emission (Draine \& Lazarian 1999), cannot be completely ruled out.

To date there have been only a handful of polarization observations of individual AME regions, all of which show evidence of little or no polarization: to the level of a few percent (Rubiño-Martin et al. 2012; Battistelli et al. 2006; Mason et al. 2009; Dickinson et al. 2007; Casassus et al. 2008; Hoang et al. 2013; Lopez-Caraballo et al. 2011; Dickinson et al. 2011). AME polarization is able to differentiate between the different 
models since different models predict a different polarization percentage. This is the case, for instance, of magnetic dipole emission, which predicts a polarization fraction that can be of the order of $10 \%$ or more with frequency-dependent behavior. In contrast, Stokes $I$ multi-band observations in the $5-30 \mathrm{GHz}$ range are essential to separate the AME from the other types of ISM emission in conjunction with millimeter observations for thermal dust removal. High angular resolution measurements are fundamental to understanding the physics behind the AME and to limit source confusion (see, e.g., Battistelli et al. 2012). In this respect, though, it is remarkable that, up until now, no observation has had sufficient sensitivity, resolution, or frequency coverage to disentangle fully the numerous candidate mechanisms. Arcminute-level angular resolution observations are crucial to disentangling different contributions within the same Galactic region. These are starting to reveal cases in which the dust-to-radio correlation is observed to decrease when we go to finer angular scales (see, e.g., Vidal et al. 2011; Castellanos et al. 2011; Tibbs et al. 2013). Ysard et al. (2011) and others have suggested that this behavior could be due to the fact that whereas the infrared (IR) dust emission is proportional to the intensity of the radiation field and to the dust column density, it is not as straightforward for spinning dust emission since its excitation processes are more numerous and complex. Indeed, the rotation of interstellar grains is both excited and damped by the emission of IR photons (proportional to the radiation field intensity), collisions with neutrals $\left(\mathrm{HI}_{\mathrm{I}}, \mathrm{H}_{2}\right)$ and ions ( $\mathrm{H}$ II, $\mathrm{C}$ II), plasma drag ( $\mathrm{H}$ II, C II), photoelectric emission, formation of $\mathrm{H}_{2}$ on the grain surface, and the emission of electric dipole radiation itself (Draine \& Lazarian 1998).

A detailed multi-wavelength study of RCW175 has been performed by Tibbs et al. (2012) based on publicly available data. Tibbs et al. (2012) computed the star formation rate of the region, identified the position of young stellar object candidates, and estimated the total dust mass of RCW175. By combining high angular resolution Spitzer (Werner et al. 2004) and Herschel (Pilbratt et al. 2010) IR data with the dust model DustEM (Compiêgne et al. 2011), Tibbs et al. (2012) characterized the dust properties within the region. They crosscorrelated the coarse angular resolution microwave data (e.g., $\mathrm{CBI}$ ) with the derived dust property maps, and found that the AME is not strongly correlated with the smallest dust grain abundance but rather with the interstellar radiation field within the region. A missing key piece of information in the available data set are observations in the range $8-25 \mathrm{GHz}$, where the AME is expected to rise. These are essential for a detailed study of the physical characteristics of the AME, as well as for separating the various types of emission.

In this paper we present high angular resolution, multifrequency, and polarization-sensitive observations of this source at $8.4,13.5$, and $21.5 \mathrm{GHz}$ conducted with the Parkes Radio Telescope. These observations cover the gap in the spectral energy density (SED) of currently available data and can increase our understanding of the morphology of RCW175 at frequencies where the AME is a significant fraction of the total emission. This paper is structured as follows: in Section 2 we describe the observations we have conducted with the Parkes telescope; in Section 3 we discuss the SED derived from our new Parkes observations in addition to other data available in literature. In Section 4 we discuss the morphology of the region, including the spatial distribution of the AME; in Section 5 we present the results obtained from cross-correlating Herschel and Spitzer IR maps with microwave maps, while in Section 6 we present the results obtained from our polarization observations. In Section 7 we discuss the interpretation of the results and present our conclusions.

\section{OBSERVATIONS AND DATA REDUCTION}

The observations were conducted at three different frequency bands with the Parkes Radio Telescope, NSW Australia, a 64 m telescope operated as a National Facility by ATNF-CASS, a division of CSIRO. Photometric observations at $8.4 \mathrm{GHz}$ and $13.5 \mathrm{GHz}$ are described in Sections 2.1 and 2.2, respectively, while the polarimetric observations at $21.5 \mathrm{GHz}$ are described in Section 2.3.

\section{1. $8.4 \mathrm{GHz}$ Observations}

The $8.4 \mathrm{GHz}$ observations were conducted with the MARS receiver of the Parkes telescope on 2011 July 26 for $4 \mathrm{hr}$. The receiver is a circular polarization system with $T_{\text {sys }} \sim 30 \mathrm{~K}$, a resolution of FWHM $=2.4$ arcmin, and a bandwidth of $400 \mathrm{MHz}$ centered at a frequency of $8.4 \mathrm{GHz}$. To detect the whole useful bandwidth, the backend Digital Filter Banks Mark 3 (DFB3) was used with a configuration bandwidth of $1024 \mathrm{MHz}$ and 512 frequency channels each of width $2 \mathrm{MHz}$.

The correlator has full Stokes parameter capability, recording the two autocorrelation products $\mathrm{RR}^{*}, \mathrm{LL}^{*}$ and the complex cross-product of the two circular polarizations $\mathrm{RL}^{*}$ whose Real and Imaginary parts are the two Stokes parameters $Q$ and $U$. The feed illuminates the primary mirror with an edge taper of $19 \mathrm{~dB}$, the first side lobe amplitude is $-30 \mathrm{~dB}$ relative to the antenna pattern peak, and the gain is $1.18 \mathrm{Jy} \mathrm{K}^{-1}$.

The flux density scale was calibrated using the source PKS B1934-638 with an accuracy of 5\% (Reynolds 1994). This source is compact compared to the beam size, and in the case of slightly extended sources, as for RCW175 at this resolution, some flux can be picked up by the first side lobes resulting in an error of the flux scale accuracy. To check this, we have compared the solid angle of the main beam and first sidelobes. Our calibration observations are not sensitive enough to measure the sidelobes, but their shape can be estimated in the case of a mirror illuminated by a Gaussian feed ${ }^{9}$ as a function of its edge taper and the central blockage of the focus cabin (Goldsmith 1987). Following Goldsmith (1987), we find that the first sidelobe stretches out to 11 arcmin (diameter), which covers most of our source in one dimension and exceeds it in the other, and its solid angle is $0.6 \%$ of the main beam. The effects on the flux accuracy are thus negligible. This confirms the usual assumption that it is safe to use a flux calibration performed using compact sources for objects extending up to a few beamwidths. The main beam efficiency is $92 \%$, consistent with the results from Goldsmith (1987) for similar edge taper values.

The channels spanning the IF were then binned into twenty $20 \mathrm{MHz}$ sub-bands for the subsequent map-making processing. A standard basket-weaving technique with orthogonal scan sets along R.A. and decl. spaced by 45 arcsec was used to observe an area of $30^{\prime} \times 30^{\prime}$ centered on the source. The scan speed was 0.5 minute $^{-1}$ with a sampling time of $0.25 \mathrm{~s}$. Map-making software based on the Emerson et al. (1988) Fourier algorithm was applied to make the maps (Carretti et al. 2010). This technique effectively reduces $1 / f$ noise, and removes stripes and features different in the two orthogonal sets of scans.

\footnotetext{
9 A Gaussian is a good approximation of modern feed horn profiles.
} 


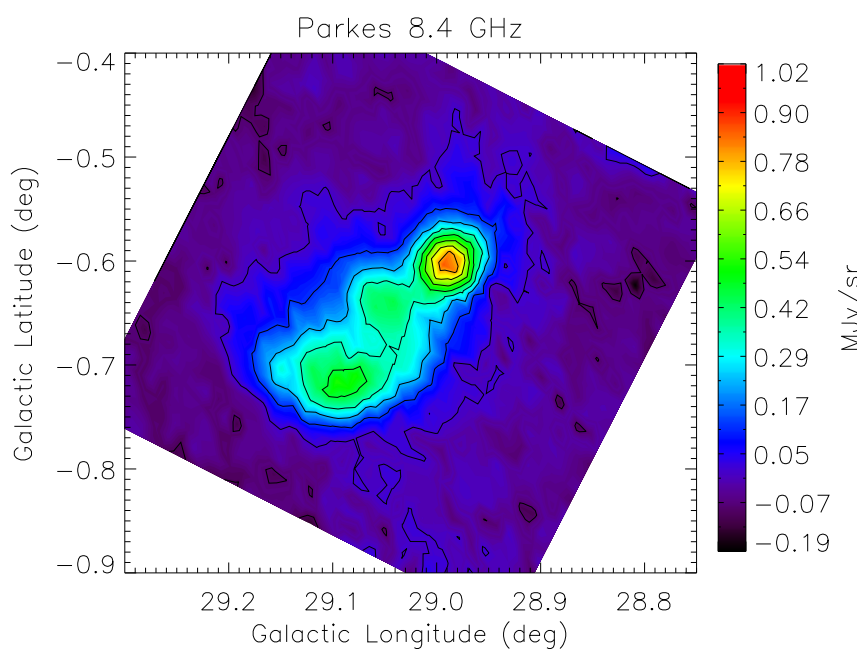

Figure 1. 8.4 GHz map of RCW175 obtained with the Parkes radio telescope using the MARS receiver. The angular resolution is 2.4 arcmin FWHM and sensitivity is $22 \mathrm{mJy}_{\text {beam }}{ }^{-1}$. The contour intervals are $10 \%$ of the peak emission.

The 20 sub-band maps were then binned together into one map for the analysis (see Figure 1). The final rms on the map is $22 \mathrm{mJy}^{\text {beam }^{-1}}$ on beam-size scales. This is higher than the expected sensitivity (some $0.7 \mathrm{mJy}$ beam $^{-1}$ ). However, $\mathrm{RCW} 175$ is close to the Galactic plane at $(l, b)=\left(29^{\circ} .1,-0.7\right)$, and such an excess rms signal might be due to diffuse Galactic emission. To investigate this option, we have used the map at $1.4 \mathrm{GHz}$ and resolution of $14.5 \mathrm{arcmin}$ of CHIPASS (Calabretta et al. 2014), where we find that an rms signal of $\mathrm{rms}_{1.4 \mathrm{GHz}}^{14.5}=$ $700 \mathrm{mK}$ in the area around RCW175. The behavior of the rms signal with the angular scale is described by rms $\propto \sqrt{\ell(\ell+1) C_{\ell}}$, where $C_{\ell}$ is the angular power spectrum and the multipole $\ell$ relates to the angular scale $\theta$ as $\theta \sim 180^{\circ} / \ell$. The angular power spectrum follows a power-law $C_{\ell} \propto \ell^{-1.7}$ near the Galactic plane (Bruscoli et al. 2002). In the $1.4-8.4 \mathrm{GHz}$ range the free-free emission is the leading contribution close to the Galactic plane with a brightness temperature that goes as $T_{b}^{\mathrm{ff}} \propto v^{-2.1}$ as a function of the frequency $v$. Rescaling the CHIPASS rms for the frequency and angular spectrum previously described, and converting the temperature rms for the calculated gain, we estimate a Galactic rms signal of $\mathrm{rms}_{8.4 \mathrm{GHz}}^{2 .}=25 \mathrm{mJy}^{2}$ beam $^{-1}$ at the frequency and resolution of our observations, consistent with the rms signal we detect around the source.

\section{2. $13.5 \mathrm{GHz}$ Observations}

The $13.5 \mathrm{GHz}$ observations were conducted with the Ku-band receiver of the Parkes telescope on 2011 August 31 and 2011 September 1 for a total of $6 \mathrm{hr}$. The receiver is a linear polarization system with $T_{\text {sys }} \sim 150 \mathrm{~K}$, a resolution of FWHM $=$ 1.7 arcmin, and a bandwidth of $700 \mathrm{MHz}$ centered at a frequency of $13.55 \mathrm{GHz}$. To detect the whole useful bandwidth, the backend Digital Filter Banks Mark 3 (DFB3) was used with a configuration bandwidth of $1024 \mathrm{MHz}$ and 512 frequency channels ( $2 \mathrm{MHz}$ each). Only the two autocorrelation products were used $\left(X X^{*}, Y Y^{*}\right)$ for Stokes $I$ measurements. The feed illuminates the primary mirror with an edge taper of $18 \mathrm{~dB}$, the first sidelobe amplitude is $-27 \mathrm{~dB}$ relative to the antenna pattern peak, and the gain is $1.55 \mathrm{Jy} \mathrm{K}^{-1}$.

The flux density scale was calibrated using the source PKS B1934-638 with an accuracy of 5\% (Reynolds 1994). As for the

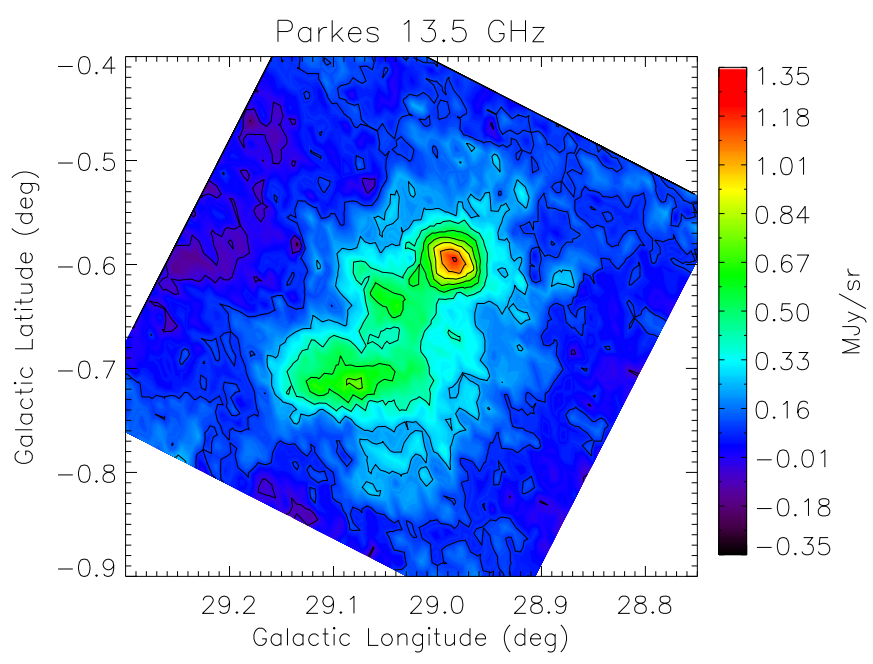

Figure 2. $13.5 \mathrm{GHz}$ map of RCW175 obtained with the Parkes radio telescope using the $K u$-band receiver. The angular resolution is 1.7 arcmin FWHM and sensitivity is $18 \mathrm{mJy}^{\text {beam }}{ }^{-1}$. Contours show levels each ranging $10 \%$ of the peak emission.

8.4 GHz data set, we could not measure the secondary lobes, and to estimate the error in the flux scale accuracy for our extended source, we estimated the antenna pattern following Goldsmith (1987). In this case, to cover most of the source, the first two sidelobes have to be considered that stretch out to $13 \mathrm{arcmin}$ (diameter). We found that their combined solid angle is $1.9 \%$ of the main beam with marginal effects on the flux scale accuracy. The main beam efficiency is $90 \%$.

The flux calibration also accounts for the atmospheric opacity attenuation. The opacity, when observing the calibrator, was $0.085 \mathrm{~dB}$ at the observing Elevation (EL); that way the flux calibration applies a constant correction for $0.085 \mathrm{~dB}$ attenuation. During the observations the opacity ranged from 0.064 to $0.120 \mathrm{~dB}$ (including EL effects) for a maximum variation compared to the constant opacity correction of $0.035 \mathrm{~dB}(0.8 \%)$, with marginal effects on the flux density scale accuracy. The opacity at the observing EL was computed from the zenithal opacity correcting for EL effects $(1 / \cos (\mathrm{EL}))$, while zenithal opacity was computed from atmospheric parameters (temperature, pressure, and relative humidity).

The channels spanning the IF were then binned into seven $100 \mathrm{MHz}$ sub-bands for the subsequent map-making processing (see Figure 2). A standard basket-weaving technique with orthogonal scan sets along R.A. and decl., spaced by 30 arcsec, was used to observe an area of $30^{\prime} \times 30^{\prime}$ centered at the source. The scan speed and sampling time were the same as those for the $8.4 \mathrm{GHz}$ observations. The same map-making software was applied to make the maps, and finally the seven sub-band maps were binned together into one. The final rms on the map is $18 \mathrm{mJy}$ beam $^{-1}$ on beam-size scales, larger than the expected sensitivity $\left(4 \mathrm{mJy}_{\text {beam }}{ }^{-1}\right)$. Following the procedure described in Section 2.1, we estimate the Galactic emission contribution at $\mathrm{rms}_{13.5 \mathrm{GHz}}^{1.7}=13 \mathrm{mJy}^{1}$ beam $^{-1}$ at the frequency and resolution of the $K u$-band observations, consistent with the observed rms.

\section{3. $21.5 \mathrm{GHz}$ Observations}

The $21.5 \mathrm{GHz}$ observations were conducted with the $13 \mathrm{~mm}$ receiver of the Parkes telescope on 2011 August 30 for a total of $4 \mathrm{hr}$. The receiver covers a band of $16-26 \mathrm{GHz}$. We used the circular polarization setup with a band of $900 \mathrm{MHz}$ centered 
at $21.55 \mathrm{GHz}$. The system temperature was $T_{\text {sys }} \sim 95 \mathrm{~K}$, and the resolution FWHM $=67$ arcsec. To detect the whole useful bandwidth, the backend Digital Filter Banks Mark 3 (DFB3) was used with a configuration bandwidth of $1024 \mathrm{MHz}$ and 512 frequency channels ( $2 \mathrm{MHz}$ each). All autocorrelation and complex cross-products of the two circular polarizations were recorded $\left(\mathrm{RR}^{*}, \mathrm{LL}^{*}, \mathrm{RL}^{*}\right)$. The feed illuminates the primary mirror with an edge taper of $21 \mathrm{~dB}$, the first sidelobe amplitude is $-30 \mathrm{~dB}$ relative to the antenna pattern peak, and the gain is $1.70 \mathrm{Jy} \mathrm{K}^{-1}$. Observations consisted of 112 repeated scans of one strip along the major dimension of the source from (R.A., decl. $)=(281.50,-3.82)$ deg to (R.A., decl. $)=(281.85,-3.68)$ deg and back (see Figure 4). This setup was aimed at detecting polarized emission at low polarization fraction or to set a new relevant upper limit able to constrain the anomalous microwave emission model space.

The flux density scale was calibrated using the source PKS B1921-293 with an assumed flux of $16.5 \mathrm{Jy}$ and accuracy of $10 \%$. This is a variable source on a timescale of a few weeks, and its flux density was measured with the Australia Telescope Compact Array the day after the Parkes observations on 2011 August 31. As for the $8.4 \mathrm{GHz}$ data, we could not measure the secondary lobes, and to estimate the error in flux accuracy for our extended source, we estimated the antenna pattern following Goldsmith (1987). The source is mostly covered by the first three sidelobes, which extend to 12 arcmin (diameter). We found their combined solid angle to be $2.8 \%$ of the main beam with marginal effects on the flux scale accuracy. The main beam efficiency is $88 \%$.

As in the case of the $13.5 \mathrm{GHz}$ observations, we have also included the atmospheric opacity attenuation in the flux calibration uncertainty. The opacity when observing the calibrator was $0.47 \mathrm{~dB}$ at the time and EL of the observation, and therefore the flux calibration applies a constant correction for $0.47 \mathrm{~dB}$ attenuation. During the observations the opacity ranged from 0.35 to $0.69 \mathrm{~dB}$ (including EL effects) for a maximum variation compared to the constant opacity correction applied of $0.22 \mathrm{~dB}$ $(5 \%)$. These, combined with the calibration uncertainty, degrade the total accuracy to $11 \%$.

On-axis instrumental polarization of the system over the observed band was $1.5 \%$. This was calibrated and corrected with observations of the planet Saturn. The procedure followed is standard: the fractional instrumental polarization on Stokes $Q$ and $U$ is measured as the fractional polarized response to an unpolarized source (Saturn in our case); then, for each piece of data to clean, the same fraction of Stokes $I$ is subtracted from the measured $Q$ and $U$. Estimate and correction have been done on each $2 \mathrm{MHz}$ frequency channel. After correction, the residual instrumental polarization on our data set was $0.2 \%$. Offaxis instrumental polarization can be as high as $0.6 \%$. However, thanks to the on-axis Parkes configuration, to the particular low polarized and diffuse observed source, and to the fact that we have performed one-dimensional scans with angles ranging from $60^{\circ}$ to $-20^{\circ}$ with respect to the horizon, cancellation effects apply to the residual contamination (see, e.g., Carretti et al. 2004; O'Dea et al. 2007). Geometrical considerations accounting for the rotation of the observed field during the observational night drive us to an estimate of a possible averaged residual polarization of $0.2 \%$. This (rather pessimistic) result drives our overall instrumental polarization uncertainty to $0.3 \%$.

The frequency channels over the $900 \mathrm{MHz}$ IF band were binned in 90 sub-bands for flux and instrumental polarization calibration. All of the sub-bands were then combined into one for the subsequent analysis. Scan length, rate, and sampling time was as for the observations at $8.4 \mathrm{GHz}$. We reached a sensitivity per beam-sized pixel of $\sigma_{Q, U}^{21.5 \mathrm{GHz}}=0.2 \mathrm{mJy}^{\text {beam }}{ }^{-1}$ in polarization, consistent with the expected value. The fluctuations in Stokes $I$ are larger with an rms of $5.4 \mathrm{mJy} \mathrm{beam}^{-1}$. This is consistent with the rms of the Galactic signal that, following the same procedure of Section 2.1, we can estimate at $\mathrm{rms}_{21.5 \mathrm{GHz}}^{67^{\prime \prime \prime}}=5.6 \mathrm{mJy}^{\text {beam }^{-1}}$ at the frequency and resolution of these observations.

It is worth noticing that the rms larger than the expected statistical noise is well accounted by the Galactic emission fluctuations at all three frequencies. Another possible source of departure from the expected statistical $\mathrm{rms}$ is the $1 / f$ noise. This is drastically mitigated by the map-making procedure, and the behavior of the additional noise we measure (decreasing with frequency) is opposite to what we would expect from this type of noise. This, along with the Galactic emission accounting for the measured rms, makes a possible contribution of the $1 / f$ noise a minor term of the error budget of our observations.

\section{SPECTRAL ENERGY DENSITY}

\subsection{Radio and Microwave Ancillary Data}

Publicly available ancillary data have been analyzed and combined with our Parkes observations to derive the SED of RCW175. At $1.4 \mathrm{GHz}$ we have considered the NRAO VLA Sky Survey (NVSS; Condon et al. 1998) data, characterized by a relatively high angular resolution $(\mathrm{FWHM}=0.75 \mathrm{arcmin})$, although they lack sensitivity on scales larger than $15 \mathrm{arcmin}$. Effelsberg $1.4 \mathrm{GHz}$ data by Reich et al. (1990a) are also available, ${ }^{10}$ but these need to be re-analyzed using the Stockert 1.4 GHz data (Reich 1982) to add missing large-scale emission to the Effelsberg data. This results, for the present analysis, in an underestimation of the integrated flux estimate form the source, and thus it has not been included in our analysis. We have thus analyzed and used, to constrain the integrated $1.4 \mathrm{GHz}$ emission, the Green Bank $300 \mathrm{ft}$ data by Altenhoff et al. (1970), which are affected by a larger uncertainty but better match our angular resolution and map size (FWHM $\approx 10$ arcmin). Data from the Effelsberg $2.7 \mathrm{GHz}$ (Reich et al. 1990b) and Parkes $5 \mathrm{GHz}$ (Haynes et al. 1978) surveys match the angular resolution needed for the present analysis, and thus we have included the flux density estimated by Tibbs et al. (2012) for these surveys.

In addition to these data, we performed an integrated flux density analysis on the GBT data $(8.35 \mathrm{GHz}$ and $14.5 \mathrm{GHz})$ by Langston et al. (2000), as well as the Nobeyama $10 \mathrm{GHz}$ data by Handa et al. (1987), which have been considered in the analysis of the integrated flux density for the RCW175 HII region. These data, however, are affected by significant systematics on angular scales that are important for our study. For instance, the Nobeyama data present a non-constant offset that is probably due to an inaccurate removal of the Galactic plane emission. For this reason, they are not included in our analysis. At microwave frequencies, as well as for our new Parkes data, we have also used the CBI $31 \mathrm{GHz}$ (Dickinson et al. 2009) and VSA $33 \mathrm{GHz}$ (Todorovic et al. 2010) flux density estimates. A summary of the data set used in this work is provided in Table 1.

\subsection{Millimetric, Sub-millimetric, and IR Ancillary Data}

We have analyzed the mm, sub-mm, and IR emission of RCW175 using data from the Planck satellite, the Herschel

\footnotetext{
10 http://www.mpifr-bonn.mpg.de/en/effelsberg
} 
Table 1

Integrated Fluxes and Instrumental Properties Used for the RCW175 Analysis and Spectral Energy Density Fit

\begin{tabular}{|c|c|c|c|c|c|}
\hline $\begin{array}{l}\text { Frequency } \\
(\mathrm{GHz})\end{array}$ & $\begin{array}{l}\text { Telescope/ } \\
\text { Experiment }\end{array}$ & $\begin{array}{l}\text { Ang. Res. } \\
\text { FWHM }\end{array}$ & $\begin{array}{l}\text { Flux Density } \\
\qquad(\mathrm{Jy})\end{array}$ & $\begin{array}{l}\text { Calibration } \\
\text { Uncertainty }\end{array}$ & Reference \\
\hline 1.4 & Green Bank $300 \mathrm{ft}$ & $9^{\prime} .4 \times 10^{\prime} .4$ & $6.0 \pm 1.8$ & Included & Altenhoff et al. (1970) \\
\hline 2.7 & Effelsberb $100 \mathrm{~m}$ & $4^{\prime} \cdot 3$ & $5.7 \pm 0.9$ & Included & Reich et al. (1990b) \\
\hline 5.0 & Parkes 64 m & $4^{\prime} .1$ & $4.0 \pm 0.8$ & Included & Haynes et al. (1978) \\
\hline 8.4 & Parkes $64 \mathrm{~m}$ & $2: 4$ & $4.86 \pm 0.51$ & $5 \%$ & This work \\
\hline 13.5 & Parkes $64 \mathrm{~m}$ & $1 ! 7$ & $5.71 \pm 0.62$ & $5 \%$ & This work \\
\hline 31 & CBI & $4^{\prime} .3 \times 4^{\prime} .0$ & $5.97 \pm 0.30$ & $1.3 \%$ & Dickinson et al. (2009) \\
\hline 33 & VSA & $13^{\prime} .1 \times 10^{\prime} .0$ & $7.8 \pm 1.6$ & $\lesssim 2 \%$ & Todorovic et al. (2010) \\
\hline 100 & Planck HFI & 9.59 & $3.2 \pm 2.7$ & $0.5 \%$ & Planck Collaboration (2011b) \\
\hline 143 & Planck HFI & $7 ! 18$ & $3.1 \pm 3.5$ & $0.5 \%$ & Planck Collaboration (2011b) \\
\hline 217 & Planck HFI & $4^{\prime} .81$ & $23 \pm 22$ & $0.5 \%$ & Planck Collaboration (2011b) \\
\hline 353 & Planck HFI & $4^{\prime} \cdot 70$ & $81 \pm 92$ & $1.2 \%$ & Planck Collaboration (2011b) \\
\hline 600 & Herschel SPIRE-500 & $36^{\prime \prime} .3$ & $650 \pm 130$ & $7 \%$ & SPIRE Obs. Man. (2011) \\
\hline 857 & Herschel SPIRE-350 & $24^{\prime \prime} .9$ & $1580 \pm 320$ & $7 \%$ & SPIRE Obs. Man. (2011) \\
\hline 1200 & Herschel SPIRE-250 & $18^{\prime \prime} .2$ & $4110 \pm 830$ & $7 \%$ & SPIRE Obs. Man. (2011) \\
\hline 1875 & Herschel PACS-160 & $10^{\prime \prime} 35$ & $9660 \pm 1940$ & $7 \%$ & Balog et al. (2014) \\
\hline 3000 & IRAS IRIS-100 & 4.3 & $10300 \pm 1800$ & $13.5 \%$ & Miville-Deschênes et al. (2005) \\
\hline 4286 & Herschel PACS-70 & 8.0 & $9600 \pm 1900$ & $7 \%$ & Balog et al. (2014) \\
\hline 5000 & IRAS IRIS-60 & $4{ }^{\prime} 0$ & $6740 \pm 1050$ & $10.4 \%$ & Miville-Deschênes et al. (2005) \\
\hline 12670 & Spitzer MIPS-24 & 6.0 & & $10 \%$ & Carey et al. (2009) \\
\hline
\end{tabular}

Notes. The fluxes have been estimated through aperture photometry of the available data (see text for details). Uncertainties are obtained by quadrature summation of the statistical and systematic/calibration uncertainties.

Space Observatory, the InfraRed Astronomical Satellite (IRAS), and the Spitzer Space Telescope. The first release of the Planck maps (Planck Collaboration 2011b) has been available since 2013 April and includes maps in nine frequency channels, ranging from 30 to $857 \mathrm{GHz}$. An overview of Planck products can be found in Planck Collaboration (2014b). CO-corrected Planck data (Planck Collaboration 2014c) have allowed us to place upper limits or obtain marginal detections at millimetric wavelengths from 100 to $353 \mathrm{GHz}$. For the color corrections we have integrated the fitting model in the Planck/HFI bandpasses, applied the corrections, and iterated until convergence, obtaining corrections ranging from $3 \%$ to $10 \%$ from 100 to $353 \mathrm{GHz}$. For the Herschel data, we have used the maps from the Hi-GAL project, a Herschel Open Time Key Programme (Molinari et al. 2010). Hi-GAL maps were obtained by PACS and SPIRE parallel mode observations, and cover five bands between 545 and $4300 \mathrm{GHz}$. The maps have been created using a dedicated map-making algorithm (Traficante et al. 2010; Piazzo et al. 2012), optimized for regions with high contrast, such as the Galactic plane. For IRAS, we have used the $60 \mu \mathrm{m}$ and $100 \mu \mathrm{m}$ IRIS (IRAS reprocessed, Miville-Deschênes et al. 2005 ) maps. Finally, we have used the Spitzer MIPS $24 \mu \mathrm{m}$ data from the MIPSGAL survey (Carey et al. 2009). A summary of the characteristics of the $\mathrm{mm}$, sub-mm, and IR data used in this work is given in Table 1.

\subsection{Integrated Emission Spectrum and Fit}

The integrated emission from the RCW175 H II region, presented in Figure 3, was obtained using aperture photometry with an annular radius of 12 arcmin and an estimate of background in the annular region from the $12-17$ arcmin range. Uncertainties were estimated from the map fluctuations outside the source. This procedure allows us to neglect structures larger than $\sim 15$ arcmin. Using the data set described above, we have fitted a model that includes the four emission mechanisms that we have assumed to be dominant in the microwave range for RCW175: synchrotron, free-free, spinning dust, and thermal dust emission.
The thermal dust was fitted to a minimum wavelength of $60 \mu \mathrm{m}$, using the IR, sub-mm, and $\mathrm{mm}$ data and a sum of two modified blackbodies, $S_{\nu} \propto v^{\beta} B B\left(\nu, T_{\text {dust }}\right)$, as in Tibbs et al. (2012). In this fit, upper limits or tentative detections derived in the Planck 143, 217, and $353 \mathrm{GHz}$ bands (Planck Collaboration 2011b) have also been included. The Planck $100 \mathrm{GHz}$ data point was instead included in the "low frequency" fit, in order to account for the impact of the thermal dust Rayleigh-Jeans tail. Planck data at frequencies above $353 \mathrm{GHz}$ were not considered, as we rely on the higher spatial resolution SPIRE data. From the fit, we retrieve results consistent with Tibbs et al. (2012), i.e., a cold dust population with temperature $T_{\mathrm{c}}=24.3_{-3.2}^{+2.4} \mathrm{~K}$, and a warm dust population with temperature $T_{\mathrm{w}}=50.8_{-1.4}^{+1.5} \mathrm{~K}$, having assumed a dust spectral emissivity index $\beta=2$ for both populations. The interstellar radiation field (ISRF) plays an important role in heating and exciting the smallest dust grains and PAHs. This can be quantified though the parameter $\chi_{\mathrm{ERF}}$, which can be estimated as $\chi_{\mathrm{ERF}}=\left(T_{\mathrm{c}} / 17.5 K\right)^{(4+\beta)}$. We find $\chi_{\mathrm{ERF}}=7$, which is indicative of a radiation field intensity a few times the ISRF in the solar neighborhood (Mathis et al. 1983; Ali-Haimoud et al. 2009; Planck Collaboration 2014a). The dust temperatures are slightly higher with respect to most of the AME sources, which are found to be associated mostly with colder regions (Planck Collaboration 2014a); however, they follow the same trend as found by Tibbs et al. (2011) for the Perseus complex, where the strength of the ISRF was found to be of key importance for AME regions.

The low-frequency data $(<8 \mathrm{GHz})$ were initially fitted with a single power law (i.e., $S_{v} \propto v^{\alpha}$ ), which resulted in an average spectral index of $\alpha=-0.28_{-0.06}^{+0.09}$. Later, a fit was performed by accounting for a free-free component plus a synchrotron component, with intensity and spectral index left free to vary $\left(S_{v} \propto A_{\mathrm{ff}} \nu^{\alpha_{\mathrm{ff}}}+A_{\mathrm{sy}} \nu^{\alpha_{\mathrm{sy}}}\right)$. The results show that, below $8 \mathrm{GHz}$, the emission is dominated by free-free emission with spectral index $\alpha_{\mathrm{ff}}=-0.18 \pm 0.07$, with an additional sub-dominant contribution due to a steeper power-law with spectral index $\alpha_{\text {sy }}=-0.7_{-0.2}^{+0.5}$, consistent with synchrotron emission. At $1 \mathrm{GHz}$, the fraction of free-free emission with respect to 


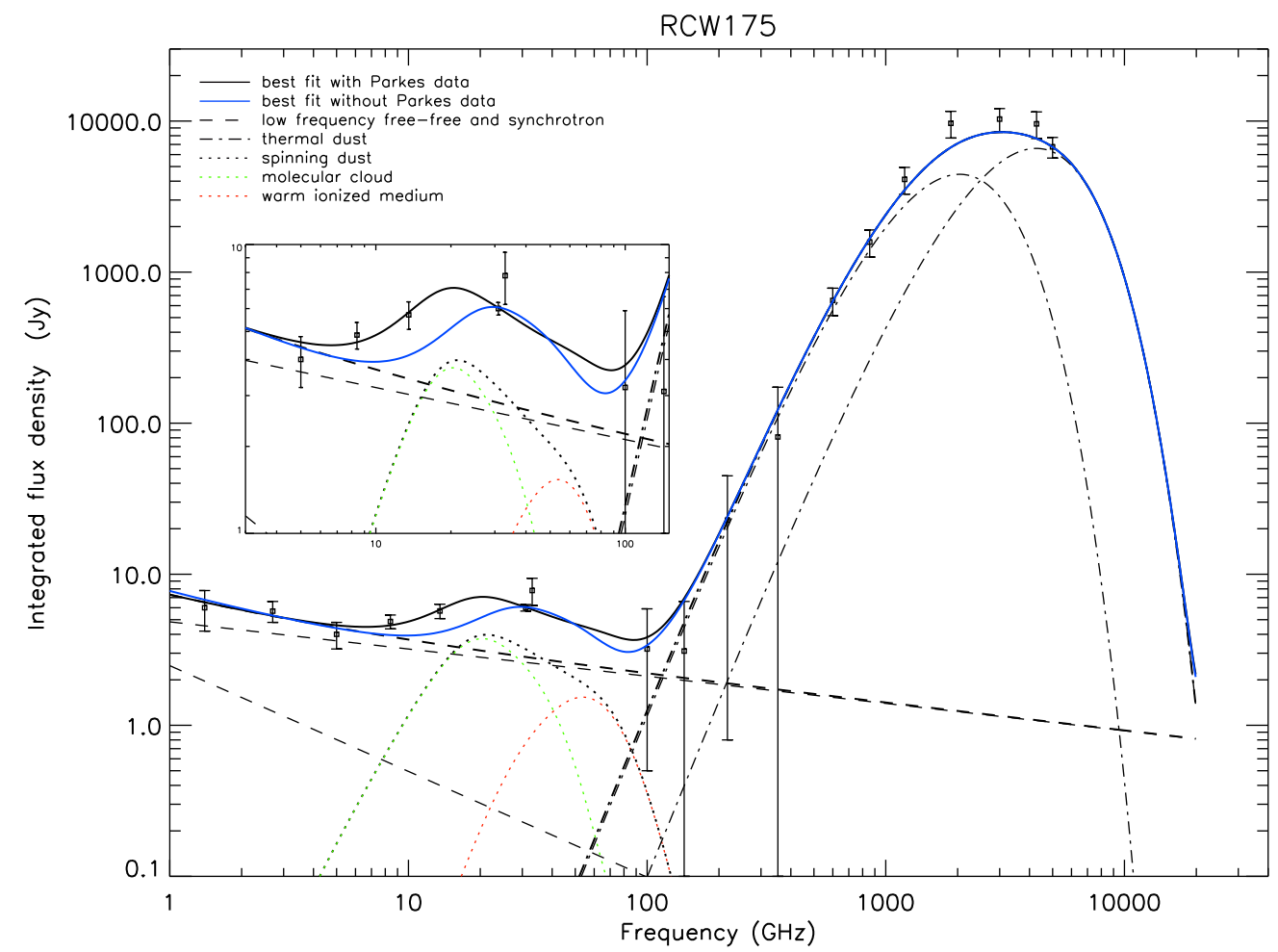

Figure 3. SED of RCW175 obtained with aperture photometry in a 12 arcmin radius. The fit is performed with free-free, synchrotron, thermal dust emission, and different spinning dust models. The black solid line denotes the sum of all the fitted components. Dashed lines indicate the low frequency (i.e., free-free and synchrotron) components, dot-dashed lines show the thermal dust fit, and dotted lines denote the spinning dust models. The blue line shows the same fit, excluding the Parkes data. The overall fit suggests the presence of at least two different components of anomalous emission. See the text and the figure legend for more details.

synchrotron emission is $A_{\mathrm{ff}} / A_{\mathrm{sy}}=1.8$, clearly increasing with frequency and indeed confirming the hypothesis of synchrotron contamination along the line of sight of RCW175 as previously found by Tibbs et al. (2012). To fit the spinning dust component, we used the SPDUST.2.01 $1^{11}$ code (Ali-Haimoud et al. 2009; Silsbee et al. 2011) to compute different emission spectra accounting for the radiation field intensity and gas temperature of this region. The hydrogen number density and gas temperature have been chosen to be consistent with standard values associated with molecular clouds (MC) and the warm ionized medium (WIM; Draine \& Lazarian 1998; Dickinson 2012). For the WIM component we have assumed hydrogen number densities $n_{\mathrm{H}}^{\mathrm{WIM}}=26.3 / \mathrm{cm}^{3}$ derived from the $5 \mathrm{GHz}$ integrated flux of RCW175 and assuming an electron temperature, $T_{\mathrm{e}}=5800 \mathrm{~K}$, following the analysis presented in Paladini et al. (2004) and Tibbs et al. (2012). As for the MC component, we built a grid of spectra with $n_{\mathrm{H}}^{\mathrm{MC}}$ varying from $n_{\mathrm{H}}^{\mathrm{MC}}=100 / \mathrm{cm}^{3}$ to $n_{\mathrm{H}}^{\mathrm{MC}}=$ $1000 / \mathrm{cm}^{3}$, with a stepsize of $25 / \mathrm{cm}^{3}$, and performed a best fit over $n_{\mathrm{H}}^{\mathrm{MC}}$. The best fit was obtained for $n_{\mathrm{H}}^{\mathrm{MC}}=150 / \mathrm{cm}^{3}$, corresponding to a minimum $\chi^{2} /$ dof $=0.78$. The major ion fractions, $[\mathrm{H}+]$ and $[\mathrm{C}+]$, are estimated as described in Ysard et al. (2011). Fits have been performed using MPFIT (Markwardt 2009) with physically reasonable priors, such as ensuring non-negative values. Attention has also been paid to ensure that the fitted parameters avoid hitting the prior limits. The parameter uncertainties were determined through Monte Carlo analysis by randomizing the data points by their $1 \sigma$ uncertainty and evaluating the scatter as the final uncertainty. In Table 2 we collect the parameters resulting from the aforementioned fits.

\footnotetext{
11 http://www.sns.ias.edu/ yacine/spdust/spdust.html
}

\subsection{SED Interpretation}

The total integrated flux SED confirms and strengthens the presence of AME in the $\mathrm{H}$ II region RCW175. The contribution from UCHII regions in RCW175 was found to be negligible by Tibbs et al. (2012). We note that repeating the low frequency fit both including and excluding the Parkes data (which fill the gap between the radio and centimetric data) provides useful information. Additional useful information could be obtained from $90-100 \mathrm{GHz}$ high angular resolution data, as these could break a possible degeneracy between AME and a strong thermal dust Rayleigh-Jeans tail at millimetric wavelengths. By excluding the Parkes data, the SED is well fitted by a single cold spinning dust component (see the blue curve in Figure 3 ). On the other hand, including the Parkes data favors a model with an additional spinning dust component consistent with the WIM, similarly to what was presented by Planck Collaboration (2011a). In the case of our measurements, this is mainly due to the fact that the rise in the SED in the microwave range (i.e., $\sim 5-30 \mathrm{GHz}$ ) occurs at lower frequencies with respect to what a single component with physically meaningful characteristics allows. As shown by Ali-Haimoud et al. (2009) and Ysard et al. (2011), the peak frequency of the spinning dust emission is mildly sensitive to the radiation field and the gas temperature, and highly sensitive to the total hydrogen number density $n_{\mathrm{H}}$. The lower $n_{\mathrm{H}}$, the lower the peak frequency, resulting in the necessity of adding a second component with low $n_{\mathrm{H}}$ to better fit the data. We should, however, stress that the modeled AME SED is strongly model dependent, with significant degeneracy between some of the parameters. We consider this result as an example of the capability of new data to break parameter degeneracies: more data, sampling the microwave frequency space at higher resolution, are necessary to solve this degeneracy, 
Table 2

Best-fit Parameters Obtained form the SED Fit of the Integrated Emission of the RCW175 H II Region

\begin{tabular}{lcc}
\hline \hline Parameter & Value & Notes \\
\hline$T_{c}$ & $24.3_{-3.2}^{+2.4} \mathrm{~K}$ & Sum of two modified black-bodies \\
$T_{w}$ & $50.8_{-1.8}^{+1.7} \mathrm{~K}$ & Sum of two modified black-bodies \\
$\beta$ & 2 & Fixed \\
$\chi_{\text {ERF }}$ & 7 & Derived from $T_{c}$ \\
$\alpha$ & $-0.28_{-0.06}^{+0.09}$ & Single power-law \\
$A_{f f}$ & $4.8_{-1.0}^{+0.9} \mathrm{Jy}$ & Sum of two power-laws \\
$\alpha_{f f}$ & $-0.18 \pm 0.07$ & Sum of two power-laws \\
$A_{s y}$ & $2.5_{-0.7}^{+0.7} \mathrm{Jy}$ & Sum of two power-laws \\
$\alpha_{s y}$ & $-0.7_{-0.2}^{+0.5}$ & Sum of two power-laws \\
$T_{e}$ & $5800 \mathrm{~K}$ & Derived from $5 \mathrm{GHz}$ integrated flux \\
$n_{\mathrm{H}}^{\mathrm{WIM}}$ & $26.3 / \mathrm{cm}^{3}$ & Derived from $5 \mathrm{GHz}$ integrated flux \\
$N_{\mathrm{H}}^{\mathrm{WIM}}$ & $\left(0.057_{-0.022}^{+0.004}\right) \times 10^{22} \mathrm{H} / \mathrm{cm}^{2}$ & \\
$n_{\mathrm{H}}^{\mathrm{MC}}$ & $150 / \mathrm{cm}^{3}$ & \\
$N_{\mathrm{H}}^{\mathrm{MC}}$ & $\left(3.8_{-0.8}^{+0.4}\right) \times 10^{22} \mathrm{H} / \mathrm{cm}^{2}$ & Range $=\left[100 / \mathrm{cm}^{3}-1000 / \mathrm{cm}^{3}\right] ;$ step $=25 / \mathrm{cm}^{3}$ \\
\hline
\end{tabular}

Notes. The two dust temperatures, $T_{\mathrm{c}}$ and $T_{w}$, were obtained from the fit of the sum of two modified blackbodies. The dust emissivity $\beta$ has been taken equal to 2 . The ISRF has been parameterized through $\chi_{\text {ERF }}$. The low frequency fit was either performed through a single power law with spectral index $\alpha$, or through the sum of two power laws of spectral indices $\alpha_{\mathrm{ff}}$ and $\alpha_{\mathrm{sy}}$, and normalization $A_{\mathrm{ff}}$ and $A_{\mathrm{sy}}$, respectively. The spinning dust models are constrained with the hydrogen number densities $n_{\mathrm{H}}$ and the column density $N_{\mathrm{H}}$ for an $\mathrm{MC}$ and a WIM component.

together with more detailed simulations and modeling. Nevertheless, the Parkes data we use here allow us to make a first attempt in this direction and clearly show that one spinning dust component is not sufficient to reproduce the observations. Combining different hydrogen number densities $n_{\mathrm{H}}$, and fitting for the column density $N_{\mathrm{H}}$, we find a best fit for a superposition of WIM and MC components with $N_{\mathrm{H}}^{\mathrm{WIM}}=\left(0.057_{-0.022}^{+0.004}\right) \times 10^{22} \mathrm{H} / \mathrm{cm}^{2}$, and $N_{\mathrm{H}}^{\mathrm{MC}}=\left(3.9_{-0.8}^{+0.4}\right) \times$ $10^{22} \mathrm{H} / \mathrm{cm}^{2}$, and with hydrogen number densities $n_{\mathrm{H}}^{\mathrm{WIM}}=$ $26.3 / \mathrm{cm}^{3}$ (fixed) and $n_{\mathrm{H}}^{\mathrm{MC}}=150 \pm 25$ (step) $/ \mathrm{cm}^{3}$, consistent with the values found by Tibbs et al. (2012). This suggests that the RCW175 emission results from different components distributed across the region. In particular, we can speculate that the WIM component is associated with the interior of the $\mathrm{H}$ II region, and the MC component with the surrounding photodissociation region (PDR).

From the ratios $N_{\mathrm{H}} / n_{\mathrm{H}}$ we derive lengths along the line of sight of the structure producing spinning dust emission of 7.1 and $84.3 \mathrm{pc}$, respectively, for the WIM and the MC components. Considering the distance to RCW175, $3.2 \times 10^{3} \mathrm{pc}$ (Tibbs et al. 2012), and the transversal size derived from our images, $\sim 20$ arcmin, we estimate a transversal physical size of $18.6 \mathrm{pc}$. This is of the same order as the line-of-sight length of the WIM component. On the other hand, the corresponding length of the MC component is slightly higher than what one would expect under the assumption of the structure having similar sizes along the line of sight and on the plane of the sky.

\section{MORPHOLOGY}

The morphology of the RCW175 H II region has been studied by Dickinson et al. (2009) and others, who have identified two sub-regions within RCW175 (G29.0-0.6 and G29.1-0.7) and built the spectrum of the whole region, as well as the spectrum of its brightest constituent (G29.0-0.6). Tibbs et al. (2012) performed a detailed multi-wavelength study of RCW175, and described G29.0-0.6 as the brighter, more dusty component, with $\mathrm{G} 29.1-0.7$ being described as the more diffuse, more

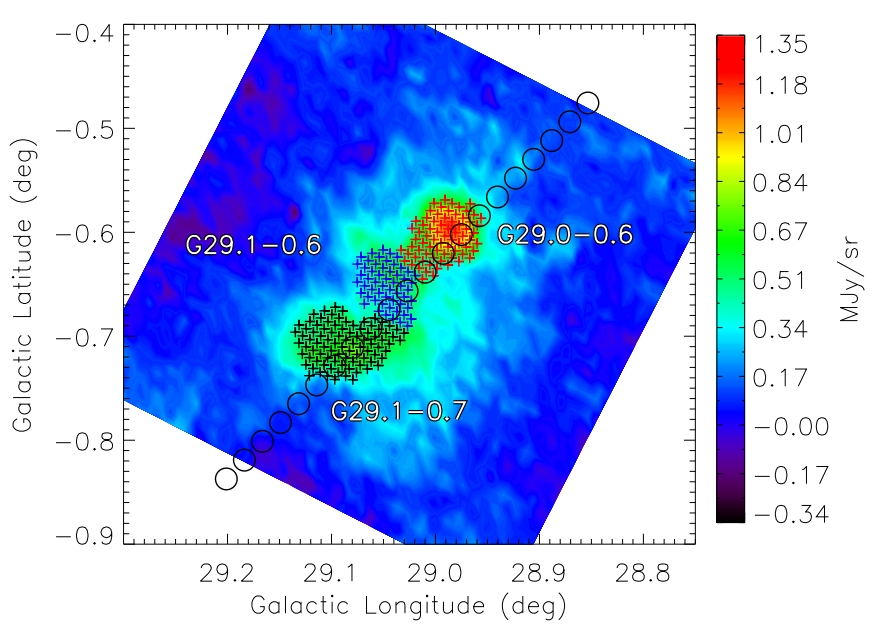

Figure 4. Parkes $13.5 \mathrm{GHz}$ map of RCW175 with the three identified sky regions highlighted. We performed our cross-correlation analysis, presented in Section 5, to the regions covered by crosses: red crosses are used to denote the compact and bright G29.0-0.6 region, blue crosses identify the G29.1-0.6 central region, and black crosses refer to G29.1-0.7. The polarization observation scan is also indicated with circles representing the size of the beam FWHM at $21.5 \mathrm{GHz}$

evolved component. So far, the only high angular resolution (that is better than 2 arcmin) maps of RCW175 have been the 1.4 GHz NVSS maps (i.e., NVSS data at $1.4 \mathrm{GHz}$ by Condon et al. 1998) at radio frequencies and, in the infrared, the Spitzer and Herschel maps. Our new microwave maps allow a more detailed study of the different spatial components of this $\mathrm{H}_{\mathrm{II}}$ region also in this frequency domain. In our maps, we can clearly identify the brighter and unresolved G29.0-0.6 region toward the west. In addition, we resolve the more diffuse G29.1 region into two separate components, i.e., G29.1-0.7 toward the east, and G29.1-0.6 toward the north (see Figure 4). A similar structure can be seen in the $1.4 \mathrm{GHz}$ NVSS data (Condon et al. 1998) and in the $10 \mathrm{GHz}$ Nobeyama map (Handa et al. 1987). 


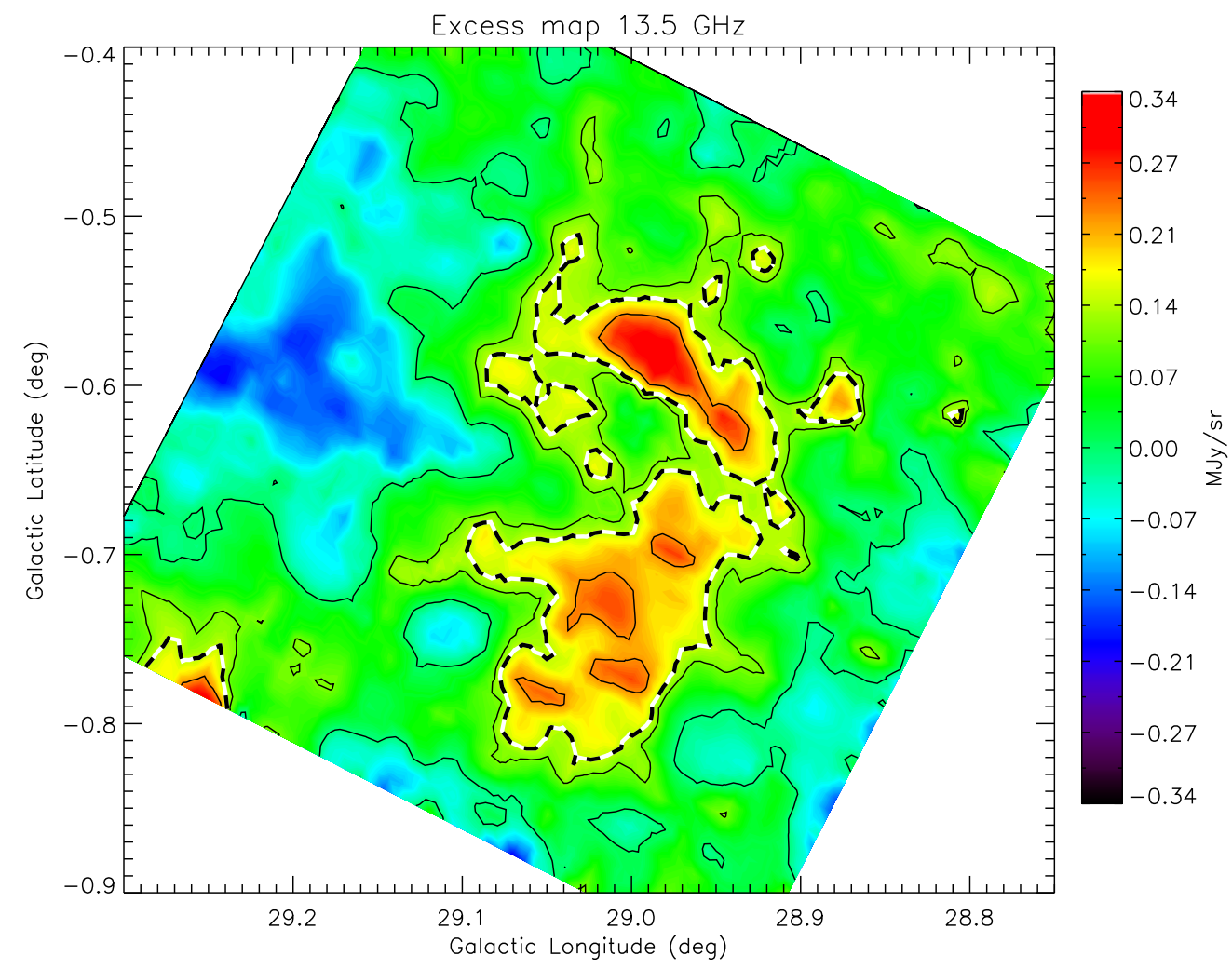

Figure 5. RCW175 map at $13.5 \mathrm{GHz}$ displaying the emission exceeding the expected level of synchrotron and free-free emission. Contours show levels separated as in Figure 1. Dashed line indicates the level corresponding to $1 \sigma$ uncertainty (see text for details).

As mentioned, with both of these data sets, we could not extract information about the larger angular scales within the region. Despite the limitations, we could probe the self-similarity of the spectral behavior of each region.

Relying on the quality and on the good (or common) systematic effect control of the Parkes maps, as well as on the self-similarity of the SED of the different constituents of the region studied, we have attempted to perform a simple component separation analysis.

To do this, we first smoothed the $13.5 \mathrm{GHz}$ map to the same angular resolution as the $8.4 \mathrm{GHz}$ map (i.e., FWHM $=$ 2.4 arcmin). We then extrapolated the $8.4 \mathrm{GHz}$ map to $13.5 \mathrm{GHz}$, assuming an average spectral index power law of $\alpha=-0.28$ (as derived in Section 3), and subtracted it from the actual $13.5 \mathrm{GHz}$ map, producing a map of the AME at $13.5 \mathrm{GHz}$ (see Figure 5). For this analysis we have assumed that the $8.4 \mathrm{GHz}$ map is not contaminated by AME, resulting in a bias in the extracted map, which should thus be treated as an underestimate of the AME in the region. The difference map clearly reveals residual emission that is mainly concentrated in the compact component, G29.0-0.6, plus an additional diffuse component surrounding the G29.1 complex. In Figure 5 we use a dashed line to illustrate the level corresponding to $1 \sigma$ uncertainty. The internal part thus exceeds the $1 \sigma$ uncertainty where we have included a $5 \%$ error arising from the modeling uncertainty. We have also included in the uncertainty the propagated error arising from the fit affecting the estimation of the flux. We emphasize that this is one of the first attempts to perform a detailed component separation at microwave wavelengths on a single galactic source of AME. This approach will likely represent a pathfinder for detailed studies of the physics of the individual ISM components.

\section{IR DATA}

A cross-correlation analysis between radio-frequency and IR data can improve our understanding of the physical components of the region. In Figures 6 and 7 we show the correlation analysis we performed using the $K u$-band $13.5 \mathrm{GHz}$ data and the SPIRE, PACS, and MIPS IR data, which span the wavelength range from $500 \mu \mathrm{m}$ to $24 \mu \mathrm{m}$. To this end, we have convolved all the maps to the $13.5 \mathrm{GHz}$ data angular resolution. On the left, we show the IR maps and, as contours, the map at $13.5 \mathrm{GHz}$; on the right, we provide the corresponding scatter plot, i.e., the IR map versus the microwave map, where different regions in the map are marked with different colors, following the same color scheme defined in Figure 4. Red crosses are used to denote the compact and bright G29.0-0.6 region, blue crosses identify the G29.1-0.6 central region, and black crosses refer to $\mathrm{G} 29.1-0.7$.

Noteworthy, the combination of sub-mm and IR bands is typically used to trace the three major populations of dust grains found in the ISM. In the reference framework of dust emission in the diffuse ISM $\left(T_{\mathrm{d}} \sim 17 \mathrm{~K}\right)$, the SPIRE $(500 \mu \mathrm{m}, 350 \mu \mathrm{m}$, and $250 \mu \mathrm{m})$ and PACS $160 \mu \mathrm{m}$ bands trace thermal dust emission from Big Grains, while the PACS $70 \mu \mathrm{m}$ and MIPS $24 \mu \mathrm{m}$ bands trace emission from very small grains (VSGs). This picture still holds (apart from the $24 \mu \mathrm{m}$ emission that we discuss below) in the case of $\mathrm{H}$ il regions.

From the $13.5 \mathrm{GHz}$ versus SPIRE-500 $\mu \mathrm{m}$ correlation, we note an absence of correlation for G29.1-0.6 and G29.1-0.7, and a mild correlation, although with a large scatter, for G29.0-0.6. A more detailed analysis reveals a shell-like structure from which the IR emission probably originates, while the microwave emission appears to generate from the bulk of 

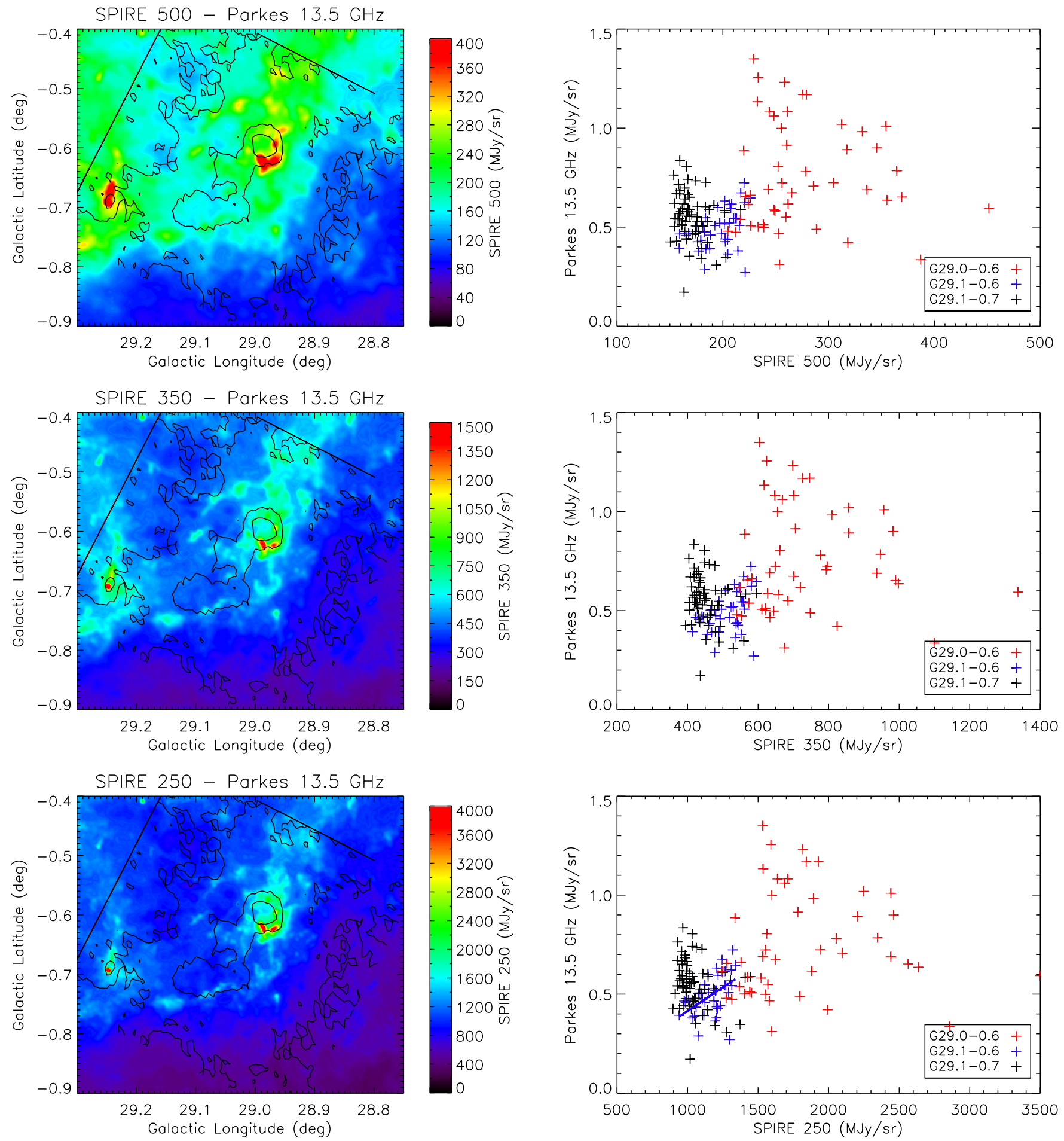

Figure 6. From top to bottom: $K u$-band $13.5 \mathrm{GHz}$ maps vs. SPIRE-500 $\mu \mathrm{m}$, SPIRE- $350 \mu \mathrm{m}$, and SPIRE- $250 \mu \mathrm{m}$ maps. The IR maps, with the $13.5 \mathrm{GHz}$ in contours (with levels at $0 \%, 33 \%$, and $66 \%$ of the peak values), are shown on the left. The corresponding scatter plots, i.e., microwave map vs. IR map, are shown on the right. Different colors identify different regions, following the color scheme in Figure 4.

G29.0-06. A similar behavior is highlighted by the correlation with the $350 \mu \mathrm{m}, 250 \mu \mathrm{m}, 160 \mu \mathrm{m}$ maps, and especially with the $70 \mu \mathrm{m}$ map.

The $13.5 \mathrm{GHz}$ versus $24 \mu \mathrm{m}$ data correlation is, at first glance, more pronounced. However, this behavior is not straightforward to interpret. As discussed by Paladini et al. (2012), the $24 \mu \mathrm{m}$ emission arising from the interior of $\mathrm{H}_{\text {II }}$ regions, contrary to what happens in the diffuse ISM, is not necessarily associated with VSG emission, but rather with BG replenishment, as proposed by Everett et al. (2010) for the case of wind-blown bubbles. Therefore, for $24 \mu \mathrm{m}$, the correlation in Figure 7 is not a priori an indication that the AME carriers are small dust grains.

In addition to the IR maps discussed above, we incorporated the dust parameter maps derived by Tibbs et al. (2012), using the DusTEM dust model (Compiêgne et al. 2011), in the correlation analysis. These parameter maps include important physical quantities such as: (1) $Y_{\mathrm{VSG}}$, the abundance of VSGs relative to 

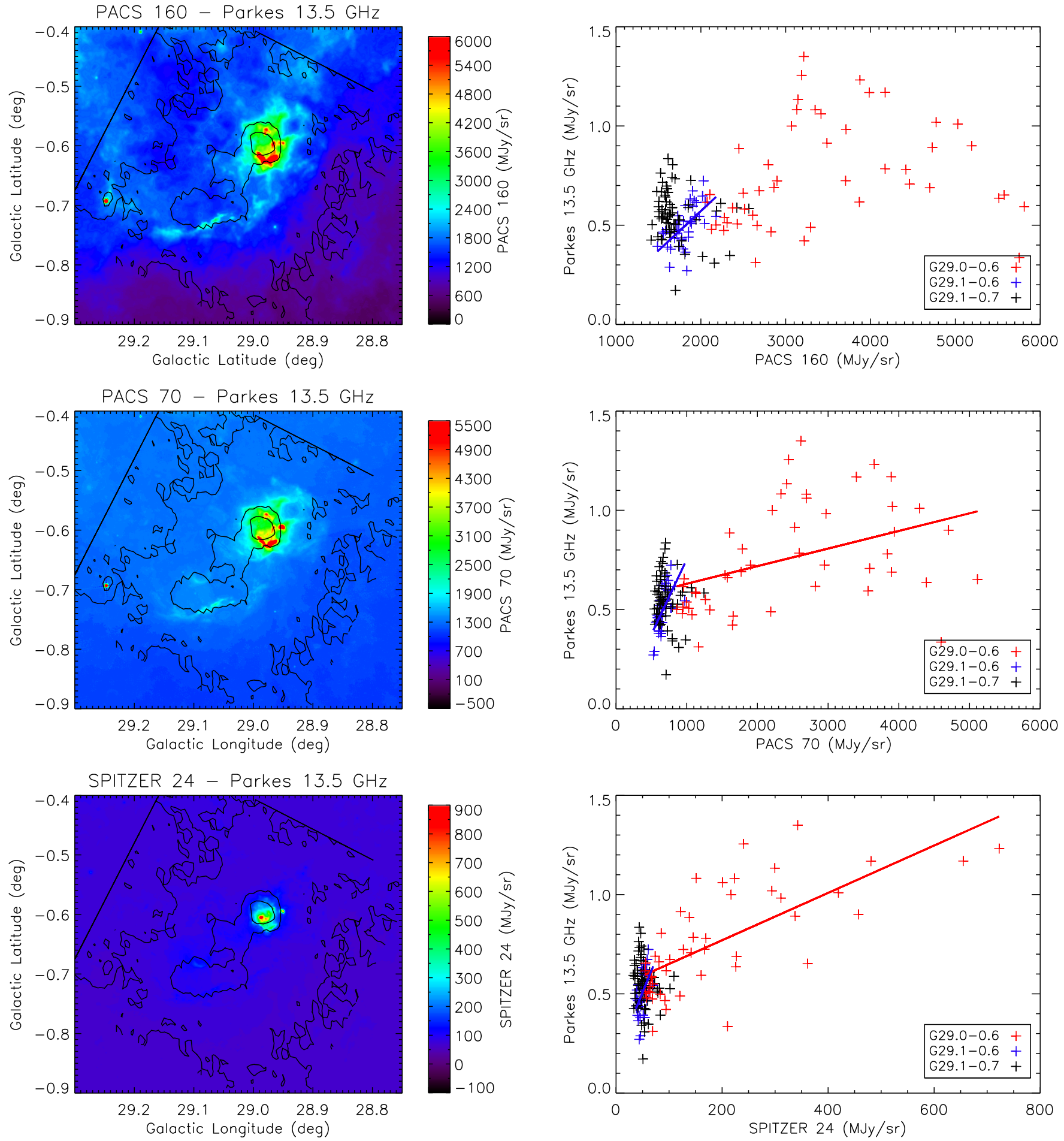

Figure 7. From top to bottom: $K u$-band $13.5 \mathrm{GHz}$ map vs. PACS- $160 \mu \mathrm{m}$, PACS-70 $\mu \mathrm{m}$, and MIPS- $24 \mu \mathrm{m}$ maps. The corresponding scatter plots, i.e., microwave map vs. IR map, are shown on the right. Different colors identify different regions, following the color scheme in Figure 4.

BGs; (2) $Y_{\mathrm{PAH}}$, the abundance of PAHs relative to BGs; (3) $\chi_{\mathrm{ERF}}$, the strength of the exciting radiation field, as parameterized in Tibbs et al. (2012); (4) $N_{\mathrm{H}}$, the hydrogen column density; and (5) $T_{\mathrm{EQ}}$, the dust equilibrium temperature derived as the median temperature of the BGs.

In Figures 8 and 9, we compare the RCW175 13.5 GHz map with the parameter maps derived from DustEM: on the left, we provide the parameter maps and, as contours, the microwave map, with all the maps at a common angular resolution; on the right, we show the corresponding scatter plot, i.e., $13.5 \mathrm{GHz}$ data versus parameter map, where, once again, different colors denote different regions of the map.

We have calculated the Pearson correlation coefficients, $\rho$, for the $13.5 \mathrm{GHz}$ map and the various IR or DusTEM parameter maps, together with the probability to exceed (PTE) that correlation value in the case of null correlation. In doing so, we have assumed that the variable $\left.t=\rho \sqrt{(}(n-2) /(1-\rho)^{2}\right)$ follows a $t$-Student distribution, with $n-2$ dof, $n$ being the number of 

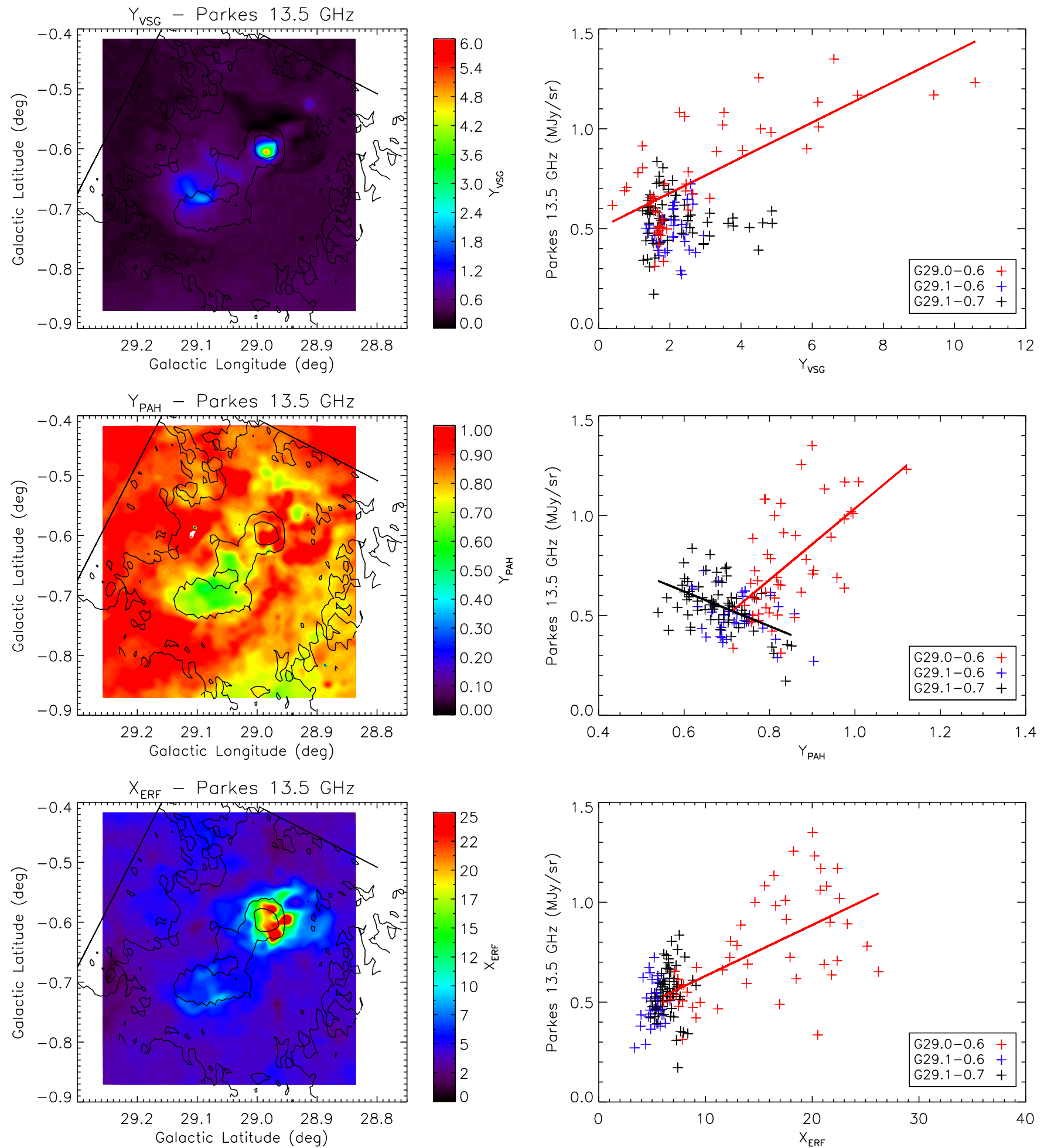

Figure 8. $K u$-band $13.5 \mathrm{GHz}$ map vs. DustEM $Y_{\mathrm{VSG}}, Y_{\mathrm{PAH}}$ and $\chi \mathrm{ERF}$ maps (top to bottom). The DustEM parameter maps, with the $13.5 \mathrm{GHz}$ in contours (with levels at $0 \%, 33 \%$, and $66 \%$ of the peak values), are shown on the left. The corresponding scatter plots, i.e., microwave map vs. parameter map, are shown on the right. Different colors identify different regions, following the color scheme in Figure 4.

data points. ${ }^{12}$ The result of this correlation measure is reported in Table 3 for the correlations between the microwave versus IR

12 The Student $t$ distribution is a very good approximation of the underlying distribution of the correlation coefficients, particularly when the correlation coefficient is zero. In this sense it can be used to test the hypothesis that the data are uncorrelated. More information on this topic can be found in Chapter 14.15 of Kendall (1952). maps, and in Table 4 for the correlations between the microwave versus DustEM parameter maps. When PTE $<0.002$, we also provide the slope of the linear fit to the microwave map versus parameter map. Microwave versus IR dust emissivities, in terms of microwave brightness temperature relative to $100 \mu \mathrm{m}$ brightness, are sometimes used to highlight common behavior of AME regions (see, e.g., Dickinson 2012). Comparison with 

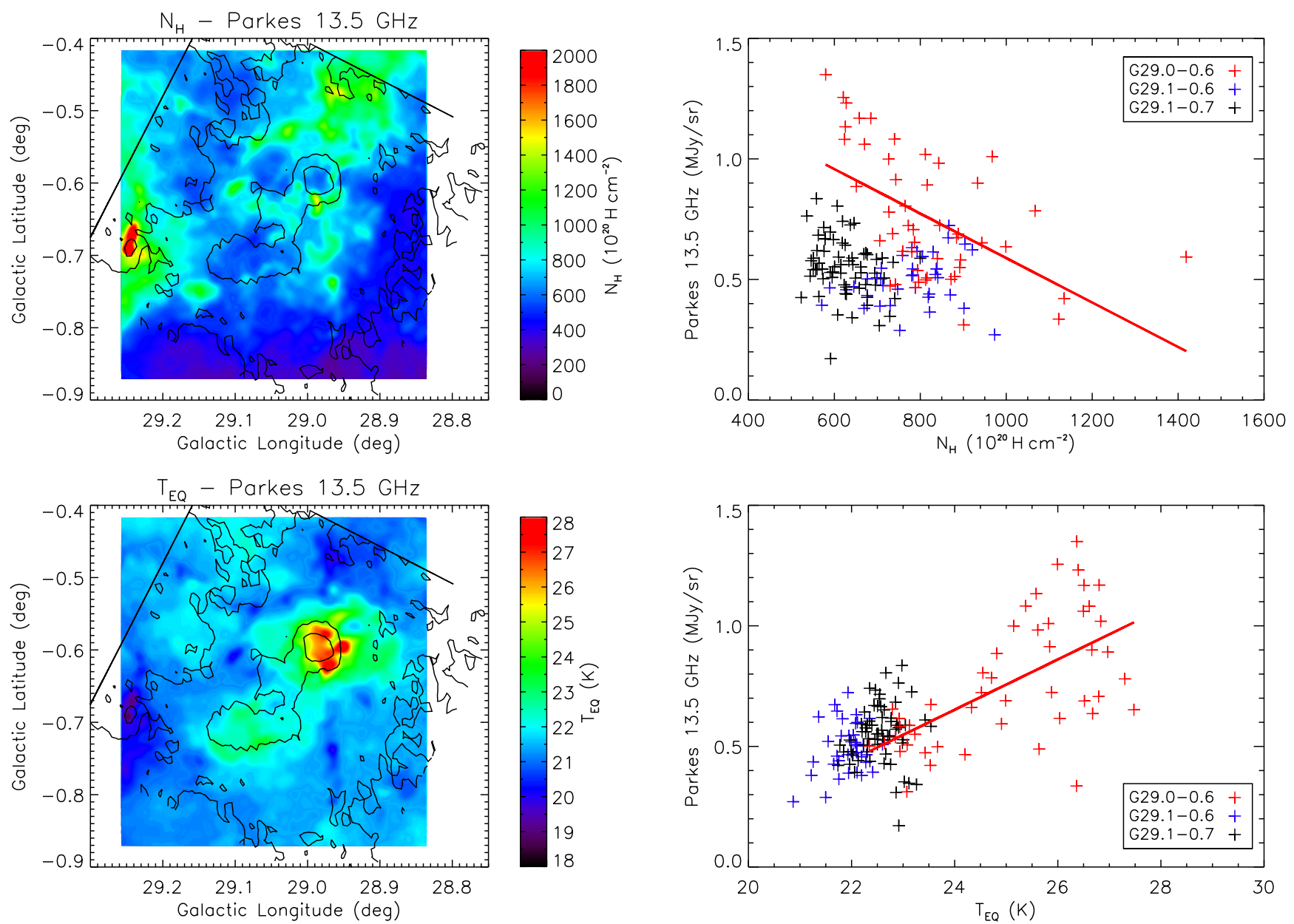

Figure 9. $K u$-band $13.5 \mathrm{GHz}$ map vs. DustEM $N_{\mathrm{H}}$ and $T_{\mathrm{EO}}$ maps (top to bottom). The DustEM parameter maps, with the $13.5 \mathrm{GHz}$ in contours (with levels at $0 \%$, $33 \%$, and $66 \%$ of the peak values), are shown on the left. The corresponding scatter plots, i.e., microwave map vs. parameter map, are shown on the right. Different colors identify different regions, following the color scheme in Figure 4.

Table 3

Summary of the Correlation Analysis of the $13.5 \mathrm{GHz}$ Map vs. the IR Maps

\begin{tabular}{lcrcc}
\hline \hline IR Map & Region & \multicolumn{1}{c}{$\rho$} & PTE & Slope \\
\hline Spitzer 24 & G29.0-0.6 & 0.70285 & 0.00000 & $(1.19 \pm 0.18) 10^{-3}$ \\
Spitzer 24 & G29.1-0.6 & 0.42984 & 0.00354 & $\ldots$ \\
Spitzer 24 & G29.1-0.7 & 0.13497 & 0.14381 & $\ldots$ \\
\hline PACS 70 & G29.0-0.6 & 0.41135 & 0.00184 & $(8.9 \pm 2.9) 10^{-5}$ \\
PACS 70 & G29.1-0.6 & 0.63511 & 0.00001 & $(8.9 \pm 7.6) 10^{-4}$ \\
PACS 70 & G29.1-0.7 & -0.05484 & 0.33345 & $\ldots$ \\
\hline PACS 160 & G29.0-0.6 & 0.21876 & 0.06761 & $\ldots$ \\
PACS 160 & G29.1-0.6 & 0.60239 & 0.00003 & $(3.98 \pm 0.88) 10^{-4}$ \\
PACS 160 & G29.1-0.7 & -0.17157 & 0.08762 & $\ldots$ \\
\hline SPIRE 250 & G29.0-0.6 & 0.02612 & 0.43006 & $\ldots$ \\
SPIRE 250 & G29.1-0.6 & 0.48720 & 0.00096 & $(4.8 \pm 1.4) 10^{-4}$ \\
SPIRE 250 & G29.1-0.7 & -0.22104 & 0.03961 & $\ldots$ \\
\hline SPIRE 350 & G29.0-0.6 & -0.03789 & 0.39911 & $\ldots$ \\
SPIRE 350 & G29.1-0.6 & 0.42209 & 0.00415 & $\ldots$ \\
SPIRE 350 & G29.1-0.7 & -0.21231 & 0.04607 & $\ldots$ \\
\hline SPIRE 500 & G29.0-0.6 & -0.03584 & 0.40445 & $\ldots$ \\
SPIRE 500 & G29.1-0.6 & 0.41731 & 0.00457 & $\ldots$ \\
SPIRE 500 & G29.1-0.7 & -0.19344 & 0.06282 & $\ldots$ \\
\hline
\end{tabular}

Notes. We provide: the Pearson correlation coefficients, $\rho$; PTE, i.e., the correlation value in the case of null correlation; at each wavelength, the slope of the linear fit to the $13.5 \mathrm{GHz}$ map versus IR map distribution. The slope is reported only when PTE $<0.002$. our correlations is not straightforward although we can see a similar qualitative behavior.

For the DustEM analysis, both the plots and Pearson coefficients reveal a clear correlation between the microwave emission in G29.0-0.6 and the dust parameters $\left(Y_{\mathrm{VSG}}, Y_{\mathrm{PAH}}, X_{\mathrm{ERF}}\right.$, and $T_{\mathrm{EQ}}$ ). The presence of highly embedded ultracompact $\mathrm{H}_{\mathrm{II}}$ regions, which could induce the observed correlations in this source component, has been ruled out by the analysis carried out by Tibbs et al. (2012). In this light, the above correlations indicate that the $13.5 \mathrm{GHz}$ emission observed in G29.0-0.6 could indeed be ascribed to AME. In the case of the G29.1 system (i.e., G29.1-0.6 and G29.1-0.7), no significant correlation is found. On the contrary, we find some indication of an anti-correlation between the microwave emission and $Y_{\mathrm{PAH}}$. This result is in apparent contradiction with the one for G29.0-0.6. Without additional information, we can only speculate that, in this case, the $13.5 \mathrm{GHz}$ emission may have an origin other than dipole emission from PAHs or VSGs.

Interestingly, our analysis highlights, for both G29.0-0.6 and the G29.1 complex, also an anti-correlation between the spatial distribution of AME and $N_{\mathrm{H}}$. Similar behavior has been observed by Lagache (2003) and Vidal et al. (2011). We can interpret this result as evidence that the bulk of AME is not generated in the PDR, where the peak of $N_{\mathrm{H}}$ is reached along the line of sight, rather in the ionized interior of the $\mathrm{H}$ II region (in G29.0-0.6) or in the surrounding diffuse 
Table 4

Summary of the Correlation Analysis of the $13.5 \mathrm{GHz}$ Map vs. the DustEM Parameter Maps

\begin{tabular}{lcrccc}
\hline \hline Parameter & Region & \multicolumn{1}{c}{$\rho$} & PTE & \multicolumn{1}{c}{ Slope } & \\
\hline$Y_{\text {VSG }}$ & G29.0-0.6 & 0.74537 & 0.00000 & $(8.8 \pm 1.2) 10^{-2}$ & $\mathrm{MJy} / \mathrm{sr}$ \\
$Y_{\text {VSG }}$ & G29.1-0.6 & 0.17953 & 0.14040 & $\ldots$ & \\
$Y_{\text {VSG }}$ & G29.1-0.7 & -0.10647 & 0.20120 & $\ldots$ & $\mathrm{MJy} / \mathrm{sr}$ \\
\hline$Y_{\text {PAH }}$ & G29.0-0.6 & 0.59550 & 0.00000 & $(1.78 \pm 0.36)$ & $\mathrm{MJy} / \mathrm{sr}$ \\
$Y_{\text {PAH }}$ & G29.1-0.6 & -0.31103 & 0.02867 & $\ldots$ & $\mathrm{MJy} / \mathrm{sr}$ \\
$Y_{\text {PAH }}$ & G29.1-0.7 & -0.49451 & 0.00002 & $(-8.7 \pm 1.9) 10^{-1}$ & \\
\hline$\chi_{\text {ERF }}$ & G29.0-0.6 & 0.57373 & 0.00001 & $(2.55 \pm 0.54) 10^{-2}$ & \\
$\chi_{\text {ERF }}$ & G29.1-0.6 & 0.22391 & 0.08829 & $\ldots$ & $\left(\mathrm{MJy} \mathrm{sr}^{-1}\right) /\left(10^{20} \mathrm{H} \mathrm{cm}{ }^{-2}\right)$ \\
$\chi_{\text {ERF }}$ & G29.1-0.7 & 0.09043 & 0.23864 & $\ldots$ & \\
\hline$N_{\mathrm{H}}$ & $\mathrm{G} 29.0-0.6$ & -0.53709 & 0.00004 & $(-9.2 \pm 2.1) 10^{-4}$ & \\
$N_{\mathrm{H}}$ & $\mathrm{G} 29.1-0.6$ & 0.24156 & 0.07200 & $\ldots$ & $\mathrm{MJy} / \mathrm{sr}$ \\
$N_{\mathrm{H}}$ & $\mathrm{G} 29.1-0.7$ & -0.24629 & 0.02489 & $\ldots$ & \\
\hline$T_{\mathrm{EQ}}$ & $\mathrm{G} 29.0-0.6$ & 0.60537 & 0.00000 & $(1.04 \pm 0.20) 10^{-2}$ & \\
$T_{\mathrm{EQ}}$ & $\mathrm{G} 29.1-0.6$ & 0.24428 & 0.06970 & $\ldots$ & \\
$T_{\mathrm{EQ}}$ & $\mathrm{G} 29.1-0.7$ & 0.10209 & 0.21107 & & \\
\hline
\end{tabular}

Notes. We provide: the Pearson correlation coefficients, $\rho$; PTE, i.e., the correlation value in the case of null correlation; for each parameter, the slope of the linear fit to the $13.5 \mathrm{GHz}$ map versus DustEM parameter map correlation. The slope is reported only when PTE $<0.002$.

medium (i.e., G29.1-0.1 and G29.1-0.7). In these regions, the abundance of PAHs and VSGs will be lower with respect to the abundance in the PDR, especially in the interior of G29.0-0.6, due to radiation pressure drift, as investigated by Draine (2011). However, our result suggests that, despite a decreased abundance, mechanisms such as ion collisions and plasma drag may supply the necessary momentum to the grains to allow them to spin, and hence emit efficiently at microwave wavelengths.

\section{POLARIZATION RESULTS}

As described in Section 2, we have performed polarization observations of RCW175 at $21.5 \mathrm{GHz}$ using one-dimensional scans through the center of the region. Our polarization measurements stand in the low signal-to-noise regime (typically $P I / \sigma<3$ ). In this case, it is well known (see, e.g., Wardle \& Kronberg 1974; Simmons \& Stewart 1985) that the determination of reliable polarized fluxes is hindered by the fact that the posterior distribution of the polarized intensity, $P I$, does not follow a normal (Gaussian) distribution. Also, PI is a quantity that must always be positive, which introduces a bias into any estimate. For any true $P I_{0}$ we would expect to measure on average a polarization $P I>P I_{0}$. In order to de-bias the measured polarized fluxes, thus obtaining an estimate of the underlying real polarization level $P I_{0}$, we follow the Bayesian approach described in Vaillancourt (2006), consisting of integrating the posterior probability density function over the parameter space of the true polarization. For $P I / \sigma<5$ we then integrate the analytical posterior shown in Vaillancourt (2006), to get the most likely de-biased polarized intensity and the associated confidence intervals at the $68.3 \%$ level, setting upper limits when $P I / \sigma<\sqrt{2}$. When $P I / \sigma \geqslant 5$, we just obtain the polarized intensity as $\sqrt{P I^{2}-\sigma^{2}}$, following Wardle \& Kronberg (1974). Vaillancourt (2006) focuses on the de-biasing of the polarized intensity, while no analytical solution is given for the posterior of the polarization fraction. Then, in order to de-bias the polarization fraction we numerically evaluate the corresponding posterior through Monte Carlo simulations, and obtain estimates of $P I / I$ or upper limits in an equivalent way as it is done for $P I$.

The de-biased estimates for the polarized signals are shown in Figure 10. In the top plot we show the polarization intensity in units of mJy beam ${ }^{-1}$, where the receiver beam at $21.5 \mathrm{GHz}$ has an FWHM of 67 arcsec. Overplotted is the intensity measured at the same frequencies. The points in the plot are separated by one beam and the errors are quoted at $2 \sigma$ with systematic uncertainty (which includes the calibration, the atmospheric opacity, and the residual instrumental polarization) linearly added in the dotted error bars. In the lower plot we show polarization fraction. Also in this second plot, the dotted error bars include the statistical and the systematic uncertainties, in this case being quantified to the level of $0.3 \%$.

The observed source appears to be weakly polarized to the level of a few percent, especially in the center of our scans. Limiting our analysis to the core of the source (i.e., for galactic latitude $28.96<\mathrm{GL}<29^{\circ} .20$ ), we find a weighted average polarized signal of $2.2 \pm 0.2$ (rand.) \pm 0.3 (sys.) $\%$ (68\% CL), where statistical uncertainties are the result of the weighted average within the core of the source and the systematic uncertainty is the residual instrumental polarization after correction.

Considering the relatively steep spectrum of the integrated low frequency emission of RCW175, it is plausible that some synchrotron contamination is present along the line of sight toward the source accounting for the relatively low level of polarization. We have modeled the presence of synchrotron radiation through the low frequency fit performed in Section 3. Assuming an average $30 \%$ polarization only for the synchrotron component, we expect up to $10 \%$ polarization of the total emission at $21.5 \mathrm{GHz}$. Also, weak polarization could be due to free-free emission in particular specific conditions of optically thick medium toward the edges of the clouds (Keating 1998). On the other hand, our result is compatible with the low level of polarization expected from electric dipole emission (i.e., $\lesssim 1 \%$ at the frequencies of our observations; Hoang et al. 2013). Multi-frequency polarization measurements would be required to discriminate whether the polarized emission comes from the synchrotron or AME component. 

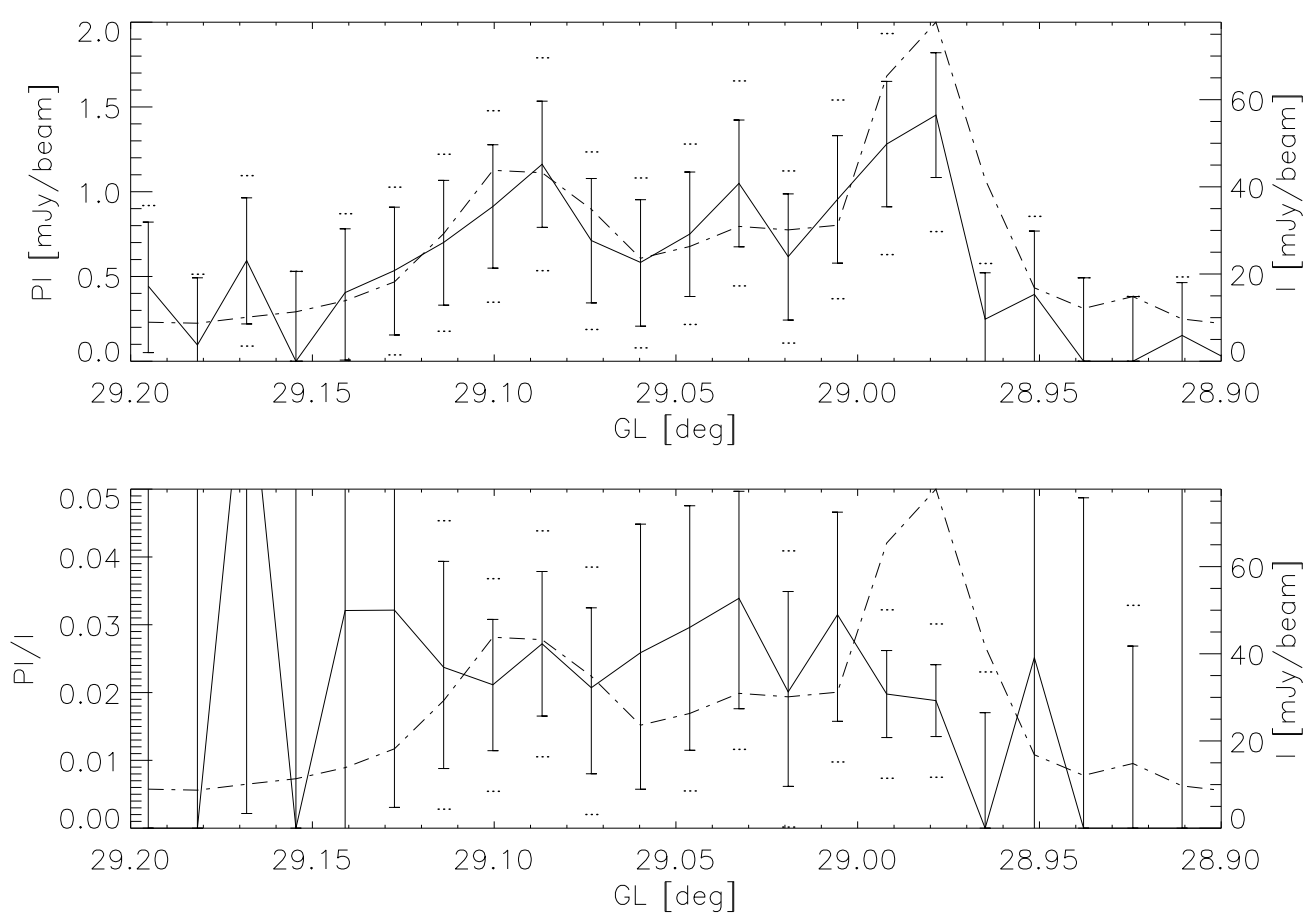

Figure 10. Linear polarization measurements performed at $21.5 \mathrm{GHz}$. For polarization measurements, one-dimensional scans have been performed on RCW175 with coordinates shown in Figure 4. The top panel displays the polarized intensity (solid line) along with an indication of the emitted intensity in arbitrary units (dot-dashed line). The error bars on $P I$ show $2 \sigma$ statistical uncertainties (solid line). The dotted bars show the linearly summed statistical uncertainties $(2 \sigma)$ to twice the systematic uncertainty (i.e., $2 \times 11 \%$ ). In the bottom panel we show, with a solid line, the relative polarized emission and, with the dot-dashed line we give an indication of the intensity emitted at $21.5 \mathrm{GHz}$. Also in this plot we include $2 \sigma$ statistical uncertainties arising from $P I$ and $I$ measurements and in the dotted bars we linearly added to the $2 \sigma$ statistical uncertainty, twice the residual instrumental polarization we qualified with $0.3 \%$. Measurements are one beam spaced.

\section{CONCLUSIONS}

We have observed the $\mathrm{H}$ II region RCW175 with the Parkes telescope at 8.4, 13.5, and 21.5 GHz. These observations confirm and strengthen the evidence for AME spatially correlated with thermal dust emission. In particular, approximately half of the RCW175 flux density in the range $15-30 \mathrm{GHz}$ appears to be due to AME, as previously reported by Dickinson et al. (2009). The SED fit suggests that multiple spinning dust components are present in the region, i.e., one associated with diffuse ionized gas, and one associated with dense molecular gas.

Combining the new Parkes observations with ancillary data, we generated an anomalous emission map at $13.5 \mathrm{GHz}$ for the RCW175 complex. The map shows that the microwave excess is characterized by both a diffuse component and a compact structure that overlaps with the location of G29.0-0.6. This corroborates the hypothesis that AME regions have a spatial extension not coincident to the bulk of microwave emission in a given region, as noted, based on statistical arguments, by Planck Collaboration (2014a).

The cross-correlation analysis of the microwave map with both the IR maps and the DUSTEM parameter maps obtained for the whole region by Tibbs et al. (2012) highlights a good degree of correlation mainly for G29.0-0.6. In this component, the investigation on small angular scales reveals an overall decrease of the microwave-IR correlation, with the exception of the $24 \mu \mathrm{m}$ map, for which the correlation is more pronounced with respect to other wavelengths. No strong correlation is found for the other $\mathrm{H}$ II region components (i.e., G29.1-0.6 and G29.1-0.7). In G29.0-0.6, the correlation with short wavelength IR data, with PAH abundance, $Y_{\mathrm{PAH}}$, with the radiation field strength, $\chi_{\mathrm{ERF}}$, and with dust temperature, $T_{\mathrm{EQ}}$, might indicate that the AME carriers are small dust grains.
However, we emphasize that the origin of the $24 \mu \mathrm{m}$ emission arising from the interior of $\mathrm{H}$ II regions is still debated.

The observed anti-correlation of $N_{\mathrm{H}}$ with the microwave map, already observed by Lagache (2003) and Vidal et al. (2011), suggests that the bulk of AME is likely emitted in low-density regions, i.e., in our case the interior of G29.0-0.6 and the diffuse medium surrounding the G29.1 complex. Additionally, it is important to keep in mind that, if the intensity of the excitation field increases, the ion fraction equally increases, leading to a higher degree of excitation for PAHs and VSGs, hence to an effective anti-correlation between microwave emission and $N_{\mathrm{H}}$. In the G29.1 complex, the prevalent diffuse emission and the mild anti-correlation between $Y_{\mathrm{PAH}}$ and the $13.5 \mathrm{GHz}$ map seem to suggest a different origin for the observed microwave excess. We emphasize that part of the $13.5 \mathrm{GHz}$, microwave excess could be due to a rising of the spectral indices between $1.4 \mathrm{GHz}$ and $5 \mathrm{GHz}$, compatible with the presence of strong stellar winds (Scaife 2012; Paladini et al. 2014) from massive OB stars. Polarization measurements at $21.5 \mathrm{GHz}$ show a low level of average polarized signal toward the center of the source of $2.2 \pm 0.2$ (rand.) \pm 0.3 (sys.) $\%$ (68\% CL) compatible with the low level of polarization of the AME or with a residual astrophysical contamination due to synchrotron or free-free from the observed region. This aspect of the analysis demands further investigations in the future.

We acknowledge the use of data from the Planck and Herschel ESA missions. Some of the results are based on observations made with the Spitzer Space Telescope, which is operated by the Jet Propulsion Laboratory, California Institute of Technology under a contract with NASA. We acknowledge the use of the HEALPix package. We acknowledge the logistic support 
provided by Parkes operators. The Parkes radio telescope is part of the Australia Telescope National Facility, which is funded by the Commonwealth of Australia for operation as a National Facility managed by CSIRO. We thank the referee for constructive comments that improved the paper.

\section{REFERENCES}

Ali-Hamoud, Y., Hirata, C. M., \& Dickinson, C. 2009, MNRAS, 395, 1055 Altenhoff, W. J., Downes, D., Goad, L., et al. 1970, A\&AS, 1, 319

AMI Consortium: Scaife, A. M. M., Hurley-Walker, N., Green, D. A., et al. 2009, MNRAS, 394, L46

Balog, Z., Müller, T., Nielbock, M., et al. 2014, ExA, 37, 129

Banday, A. J., Dickinson, C., Davies, R. D., et al. 2003, MNRAS, 345, 897

Battistelli, E. S., Carretti, E., Bernardis, P., et al. 2012, AdAst, 2012, 607384

Battistelli, E. S., Rebolo, R., Rubio-Martn, J. A., et al. 2006, ApJL, 645, L141

Bennett, C. L., Hill, R. S., Hinshaw, G., et al. 2003, ApJS, 148, 97

Bruscoli, M., Tucci, M., Natale, V., et al. 2002, NewA, 7, 171

Calabretta, M. R., Staveley-Smith, L., \& Barnes, D. G. 2014, PASA, 31, 7

Carey, S. J., Noriega-Crespo, A., Mizuno, D. R., et al. 2009, PASP, $121,875,76-97$

Carretti, E., Cortiglioni, S., Sbarra, C., et al. 2004, A\&A, 420, 437

Carretti, E., Haverkorn, M., McConnell, D., et al. 2010, MNRAS, 405, 1670

Casassus, S., Dickinson, C., Cleary, K., et al. 2008, MNRAS, 391, 1075

Castellanos, P., Casassus, S., Dickinson, C., et al. 2011, MNRAS, 411, 2, 1137

Compiêgne, M., Verstraete, L., Jones, A., et al. 2011, A\&A, 525, 103

Condon, J. J., Cotton, W. D., Greisen, E. W., et al. 1998, AJ, 115, 1693

Davies, R. D., Dickinson, C., Banday, A. J., et al. 2006, MNRAS, 370, 1125

de Oliveira-Costa, A., Kogut, A., Devlin, M. J., et al. 1997, ApJL, 482, L17

de Oliveira-Costa, A., Tegmark, M., Davies, R. D., et al. 2004, ApJL, 606, L89

de Oliveira-Costa, A., Tegmark, M., Gutiérrez, C. M., et al. 1999, ApJL,

527, L9

Dickinson, C. 2012, AdAst, 2012, 162478

Dickinson, C., Casassus, S., Davies, R. D., et al. 2010, MNRAS, 407, 2223

Dickinson, C., Davies, R. D., Allison, J. R., et al. 2009, ApJ, 690, 1585

Dickinson, C., Davies, R. D., Bronfman, L., et al. 2007, MNRAS, 379, 297

Dickinson, C., Peel, M., \& Vidal, M. 2011, MNRAS, 418, 35

Draine, B. 2011, ApJ, 723, 100

Draine, B. T., \& Lazarian, A. 1998, ApJL, 494, L19

Draine, B. T., \& Lazarian, A. 1999, ApJ, 512, 740

Emerson, D. T., \& Gräve, R. 1988, A\&A, 190, 353

Everett, J. E., \& Churchwell, E. 2010, ApJ, 713, 592

Finkbeiner, D. P., Langston, G. I., \& Minter, A. H. 2004, ApJ, 617, 350

Genova-Santos, R., Rebolo, R., Rubio-Martín, J. A., et al. 2011, ApJ, 743, 67

Goldsmith, P. F. 1987, IJIMW, 8, 771

Handa, T., Sofue, Y., Nakai, N., et al. 1987, PASJ, 39, 709

Haynes, R. F., Caswell, J. L., \& Simons, L. W. J. 1978, AuJPA, 45, 1

Hildebrandt, S. R., Rebolo, R., Rubiño-Martín, J. A., et al. 2007, MNRAS, 382,594

Hoang, T., Draine, B. T., Lazarian, A., et al. 2010, ApJ, 715, 1462

Hoang, T., Lazarian, A., Martin, P. G., et al. 2013, ApJ, 779, 152

Keating, B. 1998, ApJ, 495, 580

Kendall, M. 1952, The Advanced Theory of Statistics (London: Charles Griffin)
Kogut, A., Banday, A. J., Bennett, C. L., et al. 1996, ApJL, 464, L5

Lagache, G. 2003, A\&A, 405, 813

Langston, G., Minter, A., D’Addario, L., et al. 2000, AJ, 119, 2801

Leitch, E. M., Readhead, A. C. S., Pearson, T. J., \& Myers, S. T. 1997, ApJL, 486, L23

Lopez-Caraballo, C. H., Rubiño-Martn, J. A., Rebolo, R., Gnova-Santos, R., et al. 2011, ApJ, 729, 25

Lu, M., Dunkley, J., Page, L., et al. 2012, ApJ, 749, 165

Markwardt, C. B. 2009, in ASP Conf. Ser. 411, Astronomical Data Analysis Software and Systems XVIII, ed. D. A. Bohlender, D. Durand, \& P. Dowler (San Francisco, CA: ASP), 251

Mason, B. S., Robishaw, T., Heiles, C., Finkbeiner, D., \& Dickinson, C. 2009, ApJ, 697, 1187

Mathis, J. S., Mezger, P. G., Panagia, N., et al. 1983, A\&A, 128, 212

Miville-Deschênes, M.-A., \& Lagache, G. 2005, ApJS, 157, 302

Miville-Deschênes, M.-A., Ysard, N., Lavabre, A., et al. 2008, A\&A, 490, 1093

Molinari, S., Swinyard, B., Bally, J., et al. 2010, PASP, 122, 314

Murphy, E. J., Helou, G., Condon, J. J., et al. 2010, ApJL, 709, L108

O’Dea, D., Challinor, A., \& Johnson, B. R. 2007, MNRAS, 376, 1767

Paladini, R., Davies, R. D., De Zotti, G., et al. 2004, MNRAS, 347, 237

Paladini, R., Ingallinera, A., Agliozzo, C., et al. 2014, AAS, 223, 312

Paladini, R., Umana, G., Veneziani, M., et al. 2012, ApJ, 760, 149

Piazzo, L., Ikhenaode, D., Natoli, P., et al. 2012, ITIP, 21, 3687

Pilbratt, G. L., Riedinger, J. R., Passvogel, T., et al. 2010, A\&A, 518, 1

Planck Collaboration 2011a, A\&A, 536, A20

Planck Collaboration 2011b, A\&A, 536, A1

Planck Collaboration 2014a, A\&A, 565, A103

Planck Collaboration 2014b, A\&A, 571, A1

Planck Collaboration 2014c, A\&A, 571, A13

Reich, W. 1982, A\&AS, 48, 219

Reich, W., Fuerst, E., Reich, P., Reif, K., et al. 1990a, A\&AS, 85, 633

Reich, W., Reich, P., \& Fuerst, E. 1990b, A\&AS, 83, 539

Reynolds, J. E. 1994, ATNF Tech. Doc. Ser. 39.3040

Rubiño-Martin, López-Caraballo, C. H., Génova-Santos, R., et al. 2012, AdAst, 2012, 351836

Scaife, A. 2012, AstRv, 7, 26

Scaife, A., Green, D. A., Battye, R. A., et al. 2007, MNRAS, 377, L69

Sharpless, S. 1959, ApJS, 4, 257

Silsbee, K., Ali-Hamoud, Y., \& Hirata, C. M. 2011, MNRAS, 411, 2750

Simmons, J. F. L., \& Stewart, B. G. 1985, A\&A, 142, 100

SPIRE Observers Manual 2011, HERSCHEL-DOC-0798, version 2.4, June 7

Tibbs, C., Flagey, N., Paladini, R., et al. 2011, MNRAS, 418, 1889

Tibbs, C., Paladini, R., Compigne, M., et al. 2012, ApJ, 754, 94

Tibbs, C., Scaife, A. M. M., Dickinson, C., et al. 2013, ApJ, 768, 98

Todorovic, M. 2010, MNRAS, 406, 16291643

Traficante, A., Calzoletti, L., Veneziani, M., et al. 2010, MNRAS, 416, 2932

Vaillancourt, J. E. 2006, PASP, 118, 1340

Vidal, M., Casassus, S., Dickinson, C., et al. 2011, MNRAS, 414, 2424

Wardle, J. F. C., \& Kronberg, P. P. 1974, ApJ, 194, 249

Watson, R. A., Rebolo, R., Rubiño-Martín, J. A., et al. 2005, ApJL, 624, L89

Werner, M. W., Roellig, T. L., Low, F. J., et al. 2004, ApJS, 154, 1

Ysard, N., Juvela, M., Verstraete, L., et al. 2011, A\&A, 535, 89

Ysard, N., \& Verstraete, L. 2010, A\&A, 509, A12 